\title{
Unequal distribution of 165 mtrRNA at the 2-cell stage regulates cell lineage allocations in mouse embryos
}

\author{
Zhuxia Zheng ${ }^{1,2}$, Hongmei $\mathrm{Li}^{3}$, Qinfen Zhang ${ }^{3}$, Lele Yang ${ }^{1}$ and Huayu Qi ${ }^{1}$ \\ ${ }^{1}$ Key Laboratory of Regenerative Biology, Guangdong Provincial Key Laboratory of Stem Cell and Regenerative \\ Medicine, South China Institute for Stem Cell Biology and Regenerative Medicine, Guangzhou Institutes of \\ Biomedicine and Health, Chinese Academy of Sciences, A303, 190 Kaiyuan Boulevard, Science City, Huangpu \\ District, Guangzhou, Guangdong 510630, China, ${ }^{2}$ School of Life Science, University of Science and Technology of \\ China, Hefei 230026, China and ${ }^{3}$ State Key Lab for Bio-control, School of Life Sciences, Sun Yat-sen University, \\ Guangzhou 510275, China
}

Correspondence should be addressed to H Qi; Email: qi_huayu@gibh.ac.cn

\begin{abstract}
Cell lineage determination during early embryogenesis has profound effects on adult animal development. Pre-patterning of embryos, such as that of Drosophila and Caenorhabditis elegans, is driven by asymmetrically localized maternal or zygotic factors, including mRNA species and RNA binding proteins. However, it is not clear how mammalian early embryogenesis is regulated and what the early cell fate determinants are. Here we show that, in mouse, mitochondrial ribosomal RNAs (mtrRNAs) are differentially distributed between 2-cell sister blastomeres. This distribution pattern is not related to the overall quantity or activity of mitochondria which appears equal between 2-cell sister blastomeres. Like in lower species, $16 \mathrm{~S}$ mtrRNA is found to localize in the cytoplasm outside of mitochondria in mouse 2-cell embryos. Alterations of $16 \mathrm{~S}$ mtrRNA levels in one of the 2-cell sister blastomere via microinjection of either sense or antisense RNAs drive its progeny into different cell lineages in blastocyst. These results indicate that mtrRNAs are differentially distributed among embryonic cells at the beginning of embryogenesis in mouse and they are functionally involved in the regulation of cell lineage allocations in blastocyst, suggesting an underlying molecular mechanism that regulates pre-implantation embryogenesis in mouse.

Reproduction (2016) $151351-367$
\end{abstract}

\section{Introduction}

Embryogenesis following fertilization lays down the foundation for future animal development. During early embryogenesis, descendent of the totipotent zygote (the 1-cell embryo that formed by sperm-egg fusion) gradually lose their developmental potential and acquire specific cell fate. Understanding the molecular inner works that underlie the regulation of cell fate determination during early embryogenesis has been one of the central issues in developmental biology (Johnson 2009, Rossant \& Tam 2009, St Johnston \& Ahringer 2010, Nance 2014, Du et al. 2015).

In lower species, such as Drosophila and Caenorhabditis elegans, embryogenesis is believed being prepatterned. Unfertilized eggs and zygotes are highly polarized cells with distinctive cellular geometry, containing predisposed cell fate determinants that are asymmetrically localized in the cell, including various RNA species and RNA binding proteins (Johnstone \& Lasko 2001, St Johnston \& Ahringer 2010). Differentially segregated cell fate determinants can drive the inherited cells into specific cell lineages, such as the germ line cells (Johnstone \& Lasko 2001, Kimble \& Crittenden 2007). The early cellular polarity of eggs or zygotes can be translated into axes of developing embryos, such as the animal-vegetal and anterior-posterior axes, which are strongly correlated with the development of body axes in adult animals. Although the underpinning molecular mechanisms of these correlations remain to be fully elucidated, cell lineage formation during early embryogenesis in lower species is profoundly influenced by pre-existing intrinsic factors, mostly inherited from the maternal source.

In mammals, the establishment of distinguishable cell lineages following fertilization is thought to occur first at the blastocyst stage during pre-implantation embryogenesis (Rossant \& Tam 2009). Mammalian blastocyst initially contains two cell-lineages: the inner cell mass (ICM) and the trophectoderm (TE). With development, ICM cells are further differentiated into epiblast (Epi) that will give rise to the embryo proper and primitive endoderm (PrE) that together with the TE will generate extra-embryonic tissues, including placenta. It is postulated that, in mouse, the blastocyst is bi-laterally symmetric and can be divided into embryonic half 
(including ICM and polar TE) and abembryonic half (containing mural TE) along animal-vegetal (A-V) axis (Gardner 1997). How this bi-lateral symmetry is related to the development of body axes in adult animals is currently not understood. It is also not clear when and how different cell lineages and the axes of blastocyst are generated during early mammalian embryogenesis. Although unfertilized mouse eggs have an apparent polarity, drastic cellular changes following fertilization make it uncertain whether the maternal polarity is relevant to the cell lineage determination and axis formation in blastocyst (Whitaker 2006, Li \& Albertini 2013). Previous research showed that embryonic cells do not gain obvious morphological and molecular differences until after 8-cell stage (Ducibella \& Anderson 1975, Johnson \& Ziomek 1981, Dard et al. 2004, Guo et al. 2010). Other lines of evidences also suggested that cell lineage specification occurs only at much later stages and embryonic axis formation may be influenced by extrinsic cues, such as the geometry of the egg's extracellular coat, Zona pellucida (ZP), rather than intrinsic factors (Motosugi et al. 2005, Kurotaki et al. 2007, Tabansky et al. 2013). Data from clonal analyses and transplantation experiments in mouse, however, suggested that cells within early embryos up to 16-32-cell stages could have similar developmental potential (Fujimori et al. 2003, Tarkowski et al. 2010). One explanation for these seemingly contradictory observations is that early embryonic cells possess developmental plasticity that allows them to adapt different developmental conditions brought by both intrinsic and extrinsic stimuli (Rossant \& Tam 2009, Zernicka-Goetz et al. 2009).

Recent research in mouse have provided direct links between cell-lineage allocations in the blastocyst and early embryonic events following fertilization (Gardner 2001, Piotrowska \& Zernicka-Goetz 2001, Piotrowska et al. 2001), indicating the existence of early cell fate determinants in mammals. CDX2, the TE specific marker in the blastocyst, was found to express heterogeneously among blastomeres of 8-cell embryos when cells are starting to be allocated into either inner or outer layers (Niwa et al. 2005, Jedrusik et al. 2008). It was shown that both epigenetic modifiers (arginine methyltransferase PRMT4 and demethylase PRDM14) and histone arginine methylation (H3R26me) patterns are differentially expressed in mouse 4-cell blastomeres (Torres-Padilla et al. 2007, Burton et al. 2013). The chromatin binding dynamics of POU5F1/OCT4 has also been shown to differ among embryonic cells at 4-cell stage (Plachta et al. 2011). These earlier molecular asymmetries are functionally relevant to cell lineage allocations and axis formation in the blastocyst, reminiscent of the early cell fate determinants for mammalian embryogenesis. However, it remains to be determined whether the 4-cell stage is the earliest embryonic time when cells gain molecular variations.
First found in Drosophila, one of the maternal cell fate determinants that influence the formation of germ cells in invertebrates is the posterior mitochondrial ribosomal RNAs (mtrRNAs) that are localized outside of the organelle (Kobayashi et al. 1993, lida \& Kobayashi 1998). Examination of mtrRNAs in other species, including Xenopus and Sea urchin, also showed that they are localized asymmetrically and outside of mitochondria in early embryos, suggesting their possible functions as early cell fate determinants in these species (Kobayashi et al. 1998, Ogawa et al. 1999). Investigation of mtrRNAs in mouse pre-implantation embryos suggested that they are localized asymmetrically in the cytoplasm of MII oocytes toward the animal pole where the first polar body emits. Similar to that in lower species, $16 \mathrm{~S}$ mtrRNA was also found in the cytoplasm outside of mitochondria in MII oocytes (Ninomiya \& Ichinose 2007). However, it was suggested that mtrRNAs were absent in mouse 2-cell embryos and no difference was found in their distribution among blastomeres of preimplantation embryos. Their functional roles during cell lineage specification in mouse have yet to be determined. In the present study, we re-examined the expression and distribution patterns of mtrRNAs in mouse pre-implantation embryos. Results showed that mtrRNAs are constantly expressed during pre-implantation embryogenesis. Both small and large mtrRNAs are unequally distributed between 2-cell sister blastomeres, suggesting a molecular variation between cells at the beginning of embryogenesis in mouse. Further functional analyses suggested that alterations of levels of large subunit 16S mtrRNA in 2-cell blastomeres functionally influence cell lineage allocations in the blastocyst.

\section{Materials and methods}

\section{Animal handling and mouse embryos}

CD1 mice were used for embryo isolations for most of the experiments, except that when testing the hormonal effects on embryos, C57BL/6 mice were also used. Adult female mice (1.5to 2-month of age) were superovulated by i.p. injection of $10 \mathrm{IU}$ of pregnant mare serum gonadotropin (PMSG) per mouse, followed by $10 \mathrm{IU}$ of human chorionic gonadotropin (HCG) $48 \mathrm{~h}$ later. Injected female mice were placed with adult male mice overnight and checked for copulation plugs the following morning. Animals were sacrificed by cervical dislocation following $\mathrm{CO}_{2}$ anesthetization. Embryos at different stages were isolated in pre-warmed M2 media (Sigma M7167) and washed in KSOM media (Specialty Media, MR-020P-D). Unfertilized eggs were isolated from females without mating $16-18 \mathrm{~h}$ post HCG injection. Zygotes, early 2-cell, middle 2-cell, late 2-cell, 4-cell, 8-cell, morula and blastocyst embryos were collected 24-26 h, 31-32 h, 45-46 h, 52-53 h, 56-60 h, 67-68 h, 77-78 h and 90-92 $\mathrm{h}$ post HCG injection. As controls, CD1 mice that were naturally mated and superovulated $\mathrm{C} 57 \mathrm{BL} / 6$ mice were also used for the isolation of embryos. To isolate single blastomeres from 2-cell and 4-cell embryos, embryos were placed in Tyrode's 
solution to remove ZP and blastomeres were washed and separated by pipetting in PBS/PVP-40 ( $4 \mathrm{mg} / \mathrm{ml})$. Separated blastmeres were then transferred into PCR tubes. All animal husbandry and usage were carried out according to the guidelines of IACUC and approved by the Animal Care and Use Committee of Guangzhou Institutes of Biomedicine and Health, Chinese Academy of Sciences (Permit number: 2008023).

\section{CDNA cloning and real time quantitative RT-PCR}

cDNAs of $12 S$ and $16 S$ mtrRNA were cloned from mouse mitochondria genome extracted from CD1 mouse ovary using Mini Plasmid Extraction kit (Tiangen, Beijing, China). 12S mtrRNA cDNA was amplified using RT-PCR with a 5'-primer containing T7 promoter sequence for in vitro transcription. $165 \mathrm{mtrRNA}$ cDNA was cloned into pCIneo expression vector (Promega) between Sall/Notl sites under the T7 promoter. Plasmid pEGFP-N2 containing EGFP coding sequence was used for $\mathrm{PCR}$ and in vitro transcription of Egfp mRNA.

For the examination of $165 \mathrm{mtrRNA}$ and Actin expression in mouse pre-implantation embryos, 50 embryos at each preimplantation stage were collected from superovulated CD1 mice. Embryos of each group were lysed in $10 \mu \mathrm{l}$ lysis buffer (DEPC- $\mathrm{H}_{2} \mathrm{O}$ containing $0.1 \%$ Triton X-100, 0.1 M DTT and $1 \mu \mathrm{l}$ RNase OUT Recombinant RNase inhibitor, 5000 U, Invitrogen). Same amount of in vitro transcribed Egfp mRNA was added to each sample as internal control. The RT reaction was carried out with an adapter-oligo- $\mathrm{dT}_{24}$ primer at $50{ }^{\circ} \mathrm{C}$ for $1.5 \mathrm{~h}$. Real time quantitative PCR was done with SYBR Green Mix (Takara, Otsu Shiga, Japan) in optical 96-well reaction plates on a CFX96 Realtime System (Bio-Rad). PCRs were performed in triplicate and signals obtained were normalized against that of Egfp using the following equation: $2^{\text {(Ctegfp-CtgeneX) }}$.

Single-cell quantitative RT-PCR, expression of mitochondrial genes and the expression of $16 S \mathrm{mtrRNA}$ following microinjection were examined in the similar fashion using endogenous Actin as control. PCRs were performed in triplicate and signals obtained were normalized against that of Actin using the following equation: $2^{\text {(Ctactin-CtgeneX) }}$. Relative gene expression levels were calculated using Excel software. Primers used for cDNA cloning and qRT-PCR are summarized in Supplementary Table S1, see section on supplementary data given at the end of this article. Detailed experimental procedures are provided in Supplementary Materials and Methods.

\section{Whole-mount in situ hybridization of mouse embryos}

Briefly, digoxigenin (DIG)-labeled RNA probes were transcribed in vitro using T7 RNA polymerase (Takara) and DIG RNA Labeling Mix (Roche), and purified and stored in DEPC- $\mathrm{H}_{2} \mathrm{O}$ at $-80^{\circ} \mathrm{C}$. Collected embryos were fixed and stained with DIG-labeled cRNA antisense or sense probes $(1 \mu \mathrm{g} / \mathrm{ml}$ in pre-hybridization solution). They were then washed and incubated with anti-DIG alkaline phosphatase (AP) conjugates (Roche) at 1:2000 dilution in 1\% bovine serum albumin (BSA)/ $1 \times$ Phosphate Buffered Saline containing $0.1 \%$ Tween20 (PBST) for $2 \mathrm{~h}$. They were further washed before transferred into Staining buffer containing BCIP/NBT solution. Stained embryos were then mounted onto glass slides in 50\% glycerol/PBS and examined with an inverted microscope (Olympus IX71). ZP surrounding embryos were dissolved during hybridization. All procedures were carried out at room temperature unless indicated. Detailed experimental procedures are provided in Supplementary Materials and Methods.

Whole mount in situ hybridization (ISH) was carried out for the following genes: Actin, 12S mtrRNA, 16S mtrRNA, cytochrome b (Cytb), NADH dehydrogenase subunit $2(\mathrm{Nd} 2)$, cytochrome c-1 (Cyc1), glyceraldehyde-3-phosphate dehydrogenase (Gapdh), hypoxanthine guanine phosphoribosyl transferase (Hprt), high mobility group box3 (Hmgb3), TATA box binding protein (TBP)-associated factor 9 (Taf9) and immediate early response 5 (ler5). Primers used for amplifying probes are summarized in Supplementary Table S1.

\section{ISH at electron microscopic level}

ISH at the electron microscopic level (ISH-EM) was carried out according to the procedures described before (Yamashita et al. 2009), with some modifications. Briefly, isolated embryos were first fixed and hybridized with $16 \mathrm{~S}$ mtrRNA long cRNA probes as described above. They were then processed for thin $(100 \mathrm{~nm})$ sections cut with an Ultramicrotome (Leica Instruments, Wetzlar, Germany) and mounted onto nickel grids. Grids were subsequently stained with primary mouse monoclonal anti-DIG antibody (1:200, Sigma) and secondary goat anti-mouse IgG conjugated with 12-nm colloidal gold (1:20, Jackson ImmunoResearch, West Grove, PA, USA). Stained samples were examined using an electron microscope (Tecnai G2 Spirit, FEI). Average numbers of colloidal gold particles on sections of stained embryos were counted and calculated for average from ten frames of electron micrographs at $16800 \times$ magnification. All procedures were carried out at room temperature unless indicated. Transmission electron microscopy of thin (100 nm) embryo sections without ISH was also carried out according to the method described previously (Zhang et al. 2004). Detailed experimental procedures are provided in Supplementary Materials and Methods.

\section{Fluorescent ISH and immunofluorescent staining of mouse embryos}

Stellaris oligonuleiotide probe sets (20-mers) that cover the entire lengths of $12 \mathrm{~S}$ mtrRNA and $16 \mathrm{~S}$ mtrRNA were synthesized and tagged with Quasar 570 Dye and Quasar 670 Dye (Biosearch Technologies, Petaluma, CA, USA) respectively. Collected mouse embryos were fixed and stained with $125 \mathrm{nM}$ Stellaris probes, either singly or together. Stained embryos were then mounted onto glass slides.

For immunostaining of embryos with antibodies, embryos were fixed with $4 \%$ paraformaldehyde (PFA)/PBST $(1 \times$ PBS containing $0.1 \%$ Triton X-100), for $15 \mathrm{~min}$. After washing twice with PBST, they were blocked in $2 \%$ BSA/PBST for $2 \mathrm{~h}$ and incubated with primary antibodies for $2 \mathrm{~h}$. Fluorescently tagged secondary antibodies were then used to stain the embryos in $1 \%$ BSA/PBST for another $2 \mathrm{~h}$. Nuclei were stained with 4', 6-diamidino-2-phenylindole dihydrochloride (DAPI) $(0.5 \mu \mathrm{g} / \mathrm{ml})$ for $3 \mathrm{~min}$. Stained embryos were washed three times with PBST and mounted onto glass slides in 50\% glycerol/PBS. 
Live embryos were stained with MitoTracker Red $(200 \mu \mathrm{M}$, Molecular Probes) for detecting mitochondria content and tetramethyl rhodamine methyl ester (TMRM, $25 \mu \mathrm{M}$, Molecular Probes) for detecting mitochondrial membrane potential, with or without pre-treatment of protonophore carbonyl cyanide p-trifluoromethoxyphenyl-hydrazone (FCCP). Hoechst 33342 $(10 \mu \mathrm{g} / \mathrm{ml})$ was used for nuclei staining for $30 \mathrm{~min}$. In some cases, embryos stained with MitoTracker Red were further processed for fluorescent ISH (FISH) of mtrRNAs using Stellaris probes as described above. Embryos microinjected with $16 \mathrm{~S}$ mtrRNA sense or anti-sense RNAs, together with Egfp mRNA, were also stained with TMRM in the same way $8 \mathrm{~h}$ post-injection. Stained embryos were examined with Confocal Laser Scanning Microscopy (CLSM, LSM 710 NLO, Carl Zeiss, Jena, Germany).

Primary antibodies used were: rabbit polyclonal antiE-cadherin (Abcam, 1:50); mouse monoclonal anti-CDX2 (Abcam, ab115595, 1:50); rabbit polyclonal anti-NANOG (Abcam, ab80892, 1:100) and TRITC-Phalloidin (Sigma, 1:5000). Respective secondary antibodies used were: goat-anti-rabbit FITC conjugate (Molecular Probes, 1:1000); Goat-anti-mouse Alexa Fluor568 (Molecular Probes, 1:1000) or goat-anti-rabbit Alexa Fluor568 (Molecular Probes, $1: 1000)$. Detailed experimental procedures are provided in Supplementary Materials and Methods.

\section{RNA microinjection of 2-cell embryos and cell allocation analysis}

mRNAs of Egfp were transcribed in vitro using mMessage mMachine SP6/T7 Ultra Kit with Capping analogous (Ambion) from linearized pCS2 vector containing EGFP open reading frame (ORF) with human serum albumin $5^{\prime}$ and $3^{\prime}$-UTRs. Full-length $16 S$ mtrRNA sense or antisense RNAs were transcribed in vitro using $\mathrm{T} 7$ promoter with the same kit without Capping analogous. Transcribed RNAs were purified using RNeasy Mini Kit (Qiagen), re-suspended in DEPC- $\mathrm{H}_{2} \mathrm{O}$ and stored at $-80^{\circ} \mathrm{C}$. Ten fragments (100-200 nt in length) of anti-sense RNAs, spanning the entire $16 S \mathrm{mtrRNA}$ sequence were prepared and mixed together for microinjection. Single blastomere of a 2-cell embryo was microinjected with either Egfp mRNA $(0.8 \mu \mathrm{g} / \mu \mathrm{l})$ alone, or together with $165 \mathrm{mtrRNA}$ sense $(1.5 \mu \mathrm{g} / \mu \mathrm{l}, 2: 1)$ or anti-sense RNAs $(1.5 \mu \mathrm{g} / \mu \mathrm{l}, 2: 1)$ respectively.

Injected embryos were cultured in vitro in KSOM for 46-48 h till blastocyst stage in a humidified incubator containing $5 \%$ $\mathrm{CO}_{2}, 37^{\circ} \mathrm{C}$. Embryos were then fixed in $4 \%$ PFA/PBST and stained with DAPI $(0.5 \mu \mathrm{g} / \mathrm{ml})$. Each embryo containing EGFPlabeled $\left(\mathrm{EGFP}^{+}\right)$cells was scanned at $2-\mu \mathrm{m}$ intervals using CLSM. Scanned images from each embryo were composed together and examined at three dimensions using ZEN 2010 software (Carl Zeiss). Total number of cells and positions of individual cells within an embryo were determined by their nuclear DAPI staining, in relation to the surrounding cells. Using ortho module of the software, cells that were located on the surface of the embryo along either one of the three axes ( $\mathrm{X}, \mathrm{Y}$ and $\mathrm{Z}$ ) were designated as TE cells, whereas cells that are located on the inside of the embryo and surrounded by other cells along all three axes were assigned as ICM cells. Positions of $\mathrm{EGFP}^{+}$cells were determined by superimposing the green fluorescence over DAPI staining. Total number of cells with either EGFP signal or CDX2 staining or both were counted in the same way. Alternatively, distributions of $\mathrm{EGFP}^{+}$progeny cells in either embryonic, abembryonic or both sides within blastocysts were examined using 3-D confocal images. Embryos were grouped into three different patterns according to the allocations of $\mathrm{EGFP}^{+}$cells within them. Pattern 1 contains $\mathrm{EGFP}^{+}$cells in the embryonic side only; pattern 2 contains $\mathrm{EGFP}^{+}$cells in both embryonic and abembryonic sides and pattern 3 contains EGFP $^{+}$cells in the abembryonic side only. Total numbers of cells with or without EGFP in either ICM or TE and patterns of embryos following microinjection were counted and calculated using Excel software. Cell numbers surveyed were summarized in Supplementary Tables, see section on supplementary data given at the end of this article.

\section{Statistical analyses}

Signal intensities of whole-mount ISH were measured against background using outlined single blastomere from light micrographs using Image-Pro 6 software. Fluorescent signals, including that of $\mathrm{FISH}$, were quantified from entire stacks of confocal sections for each sister blastomere using ImageJ software. Ratios of signal intensities ( $R=$ OD1/OD2, OD1> OD2, OD: optical density measured for each sister blastomere) between 2-cell sister blastomeres were then calculated using Excel. Statistical significances of signal variances between experimental and control groups were assessed using nonparametric Mann-Whitney test for independent samples. Alternatively, significance of variation was also examined using z-test for two samples' means. One-way ANOVA and post-hoc Turkey test were performed to assess the significance of differences when there were more than two groups of data in the experiment using QIMacros software. $\chi^{2}$ test was performed for assessing the significance of changes of embryonic patterns following microinjection. Significance was set as $P<0.05$. Data are presented as mean \pm s.D.

\section{Results \\ Expression of mtrRNAs in mouse pre-implantation embryos}

The expression of 165 mtrRNA in mouse oocytes and pre-implantation embryos following fertilization was first examined using quantitative RT-PCR. In order to have an internal control with constant quantity, same amount of in vitro transcribed Egfp mRNA was added to each RNA sample extracted from embryos at various stages before RT reaction. Quantitative RT-PCR was then used to examine the expression of $165 \mathrm{mtrRNA}$. It was found that levels of $16 \mathrm{~S}$ mtrRNA remained constant in MII oocytes, zygotes and 2-cell embryos and increased dramatically after 4-cell stage, similar to the expression pattern of Actin control (Fig. 1D). No apparent decrease of $16 \mathrm{~S}$ mtrRNA was found in 2-cell embryos.

To confirm the expression of mtrRNAs in preimplantation embryos, ISH of both small (12S) and 

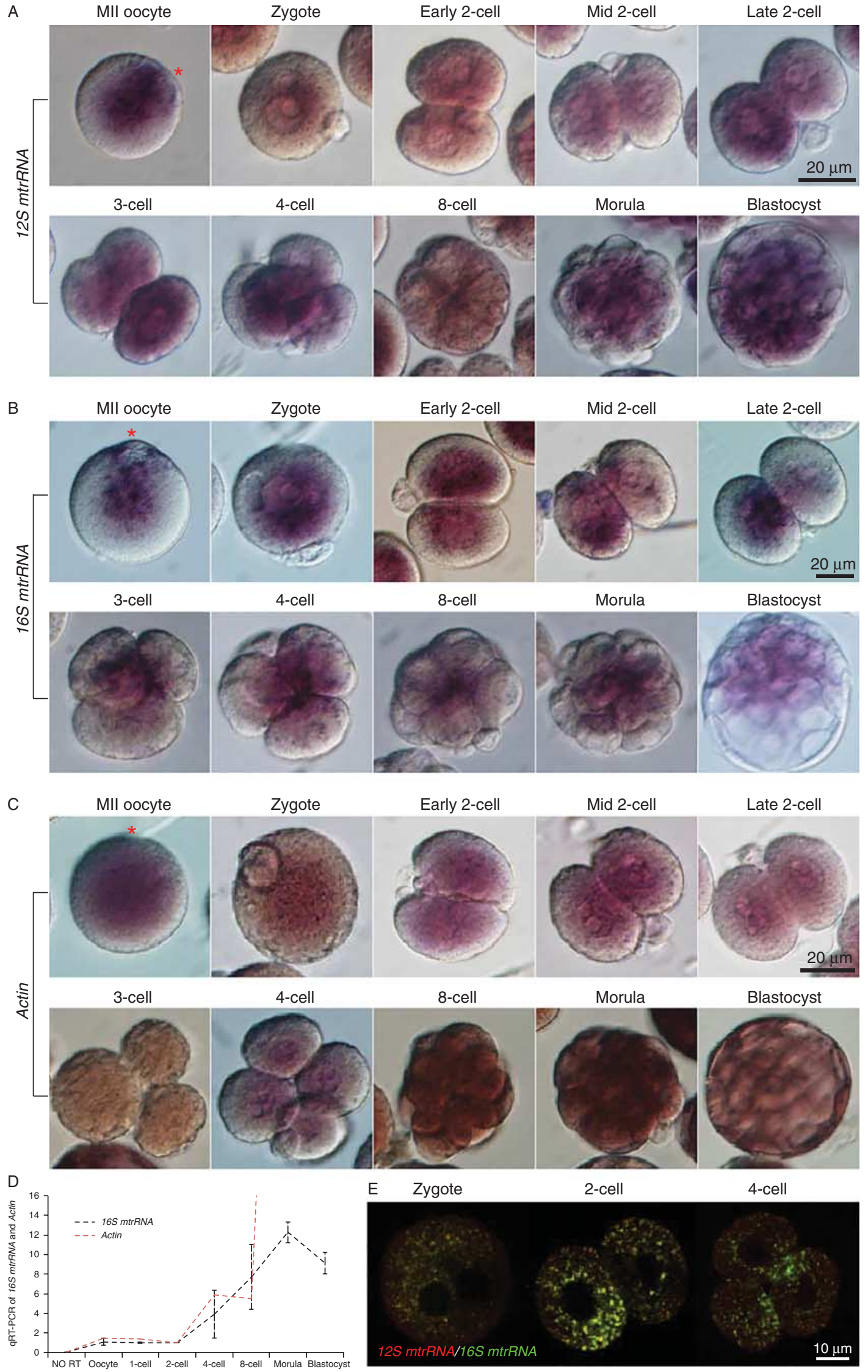
large (16S) mtrRNAs was applied using long cRNA probes containing DIG labeled uridine, coupled with anti-DIG-AP conjugates. Strong signals of both $12 \mathrm{~S}$ and $16 \mathrm{~S}$ mtrRNAs were seen in embryos at all preimplantation stages (Fig. 1A and B). In consistence with previous report, mtrRNAs were found to concentrate in the area toward the animal pole where the first polar body emits in MII oocytes (Ninomiya \& Ichinose 2007), whereas they appeared to distribute in the cytoplasm of zygotes surrounding pro-nuclei. Following compaction after 8-cell stage, higher levels of mtrRNAs were found in inner cells of morula and blastocysts, comparing with the outer cells of embryos. In contrast, Actin control appeared to be evenly distributed in all embryonic cells (Fig. 1C). Using short oligonucleotide probes (20-mers) encompassing the entire lengths of mtrRNAs, FISH was further performed to determine the expression of mtrRNAs in mouse embryos. These Stellaris oligonucleotide probes were tagged with either Quasar 570 or Quasar 670 Dye for $12 \mathrm{~S}$ and $16 \mathrm{~S}$ mtrRNAs respectively. Similarly, signals of mtrRNAs were seen in preimplantation embryos at all stages, including 2-cell and 4-cell embryos (Fig. 1E). These results indicate that mtrRNAs are continuously expressed in mouse embryos across all pre-implantation stages.

\section{mtrRNAs are differentially distributed between mouse 2-cell blastomeres}

Although no clear asymmetric distribution of mtrRNAs was found in zygotes, one of the 2-cell sister blastomeres in some embryos appeared to contain more mtrRNAs than the other (Fig. 1A and B, late 2-cells). To better quantify and define the differences of mtrRNAs between sister blastomeres, 2-cell embryos were separated into early, middle and late stages and hybridized with DIG-labeled long cRNA probes (Fig. 2A). The optical densities of ISH signals in sister blastomeres were then measured in pair for each 2-cell embryo outlined from light micrographs. Ratios of signal intensity between two sister blastomeres (the higher vs the lower one, $R=\mathrm{OD} 1 / \mathrm{OD} 2$, OD1 $>\mathrm{OD} 2)$ were calculated as an indicator for differences of RNA levels. Average ratios were obtained from groups of embryos at each 2-cell stage for each RNA species examined (Fig. 1B and C, Supplementary Table S2). It was found that the ratios of Actin mRNA signals were around 1 between sister blastomeres at all three 2-cell stages, suggesting an equal amount of Actin mRNA between sister blastomeres. In contrast, ratios of signals were much higher for both $12 S$ mtrRNA $(R=1.4 \pm 0.2, n=24$, late stage $)$ and $16 \mathrm{~S}$ mtrRNA ( $R=1.7 \pm 0.6, n=30$, late stage) between 2-cell sister blastomeres (Fig. 2B and C). The signal differences of $12 \mathrm{~S}$ and $16 \mathrm{~S}$ mtrRNAs appeared to be significantly varied when their ratios were compared with those of Actin control at the same stage $(P<0.05$, Mann-Whitney test). Furthermore, variations of mtrRNA levels between sister blastomeres increased with the development of 2-cell embryos, comparing to that of Actin $(P<0.05$, one-way ANOVA, $\alpha=0.05)$ (Supplementary Table S3). The difference of $16 \mathrm{~S}$ mtrRNA between 2-cell sister blastomeres could reach up to threefold in some of the embryos examined (Supplementary Table S2).

To confirm that the relative quantification of ISH signals indeed reflects different levels of RNA species between 2-cell sister blastomeres, additional control experiments were performed on house-keeping genes (Gapdh and Hprt) and genes that have been shown to highly express at 2-cell stage (Hmgb3, Taf9 and ler5) (Zeng et al. 2004). Unlike mtrRNAs, ratios of ISH signals for all genes examined were similar to that of Actin $(R \approx 1.1-1.2, n=29-52)$, indicating that their mRNAs are evenly distributed between 2-cell sister blastomeres (Supplementary Figure S1A and B, see section on supplementary data given at the end of this article). This also suggested that relative comparison of ISH signals could reflect the differences of RNA levels in-between cells and the mtrRNAs are indeed distributed unevenly between sister blastomeres of 2-cell embryos. Although the differences of mtrRNAs between 2-cell sister blastomeres can be as high as threefold in some embryos, the average differences of their signals between sister blastomeres are about $10-50 \%$ when their ratios of intensities were subtracted with those of Actin controls at the same stage $(13 \pm 18 \%$ for $12 S \mathrm{mtrRNA}$ and $58 \pm 67 \%$ for $16 S m t r R N A$ at late 2 -cell stage respectively). These differences (less than twofold) and large variations among samples could be difficult to reveal using PCR method due to the exponential amplification effect (Weaver et al. 2010). Nevertheless, single-cell quantitative RT-PCR of dissected blastomeres suggested similar

Figure 1 Expression of mitochondrial ribosomal RNAs in mouse pre-implantation embryos. (A) In situ hybridization of $12 S$ mtrRNA in mouse embryos at different stages. (B) In situ hybridization of $16 S \mathrm{mtrRNA}$ in mouse embryos at different stages. (C) In situ hybridization of Actin in mouse embryos at different stages. MII oocytes were also included. Stars indicate the position of the first polar body. Note the difference in signal intensities between late 2-cell blastomeres in (A) and (B). (D) Quantitative RT-PCR of $16 S$ mtrRNA in pre-implantation embryos. The expression of $16 S$ mtrRNA remained at constant level before 2-cell stage and greatly increased afterwards (black dashed line, three independent experiments). The expression of Actin also increased after 2-cell stage (red dashed line). Same amount of Egfp mRNA was added to each sample and used as internal control (see Materials and methods). PCR without RT was included as negative control. 50 embryos at each stage were used for cell lysate preparation and RT reaction. (E) Fluorescent in situ hybridization (FISH) of mouse pre-implantation embryos. Embryos were hybridized with Stellaris oligonucleotide probes tagged with Quasar 570 (for $12 S \mathrm{mtrRNA}$ ) and Quasar 670 dye (for $16 S \mathrm{mtrRNA}$ ) simultaneously. $165 \mathrm{mtr} R \mathrm{NA}$ signals were pseudo-colored in green. Shown are merged confocal images. 

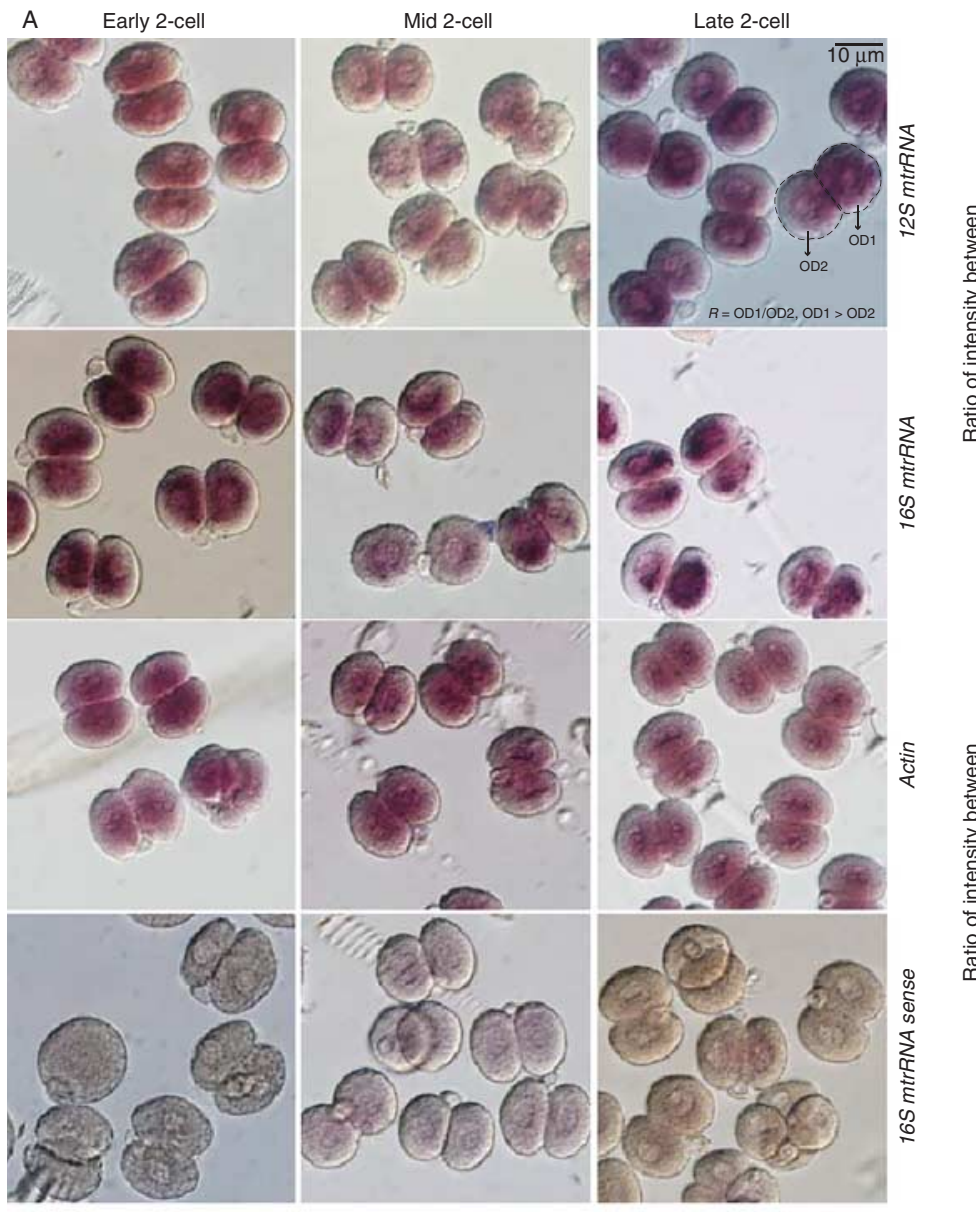

B Ratio of intensity between sister blastomeres
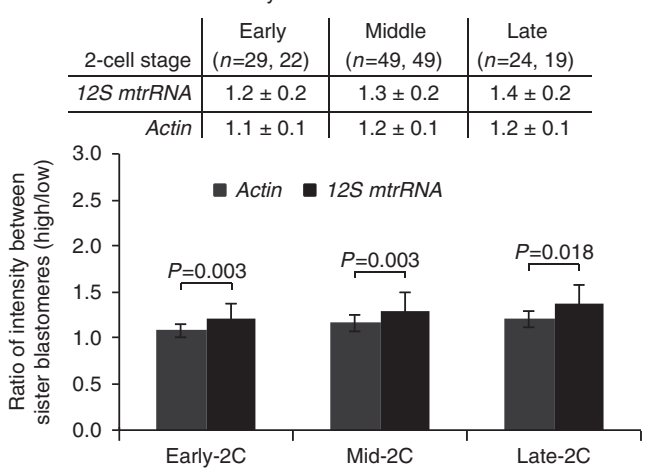

C Ratio of intensity between sister blastomeres

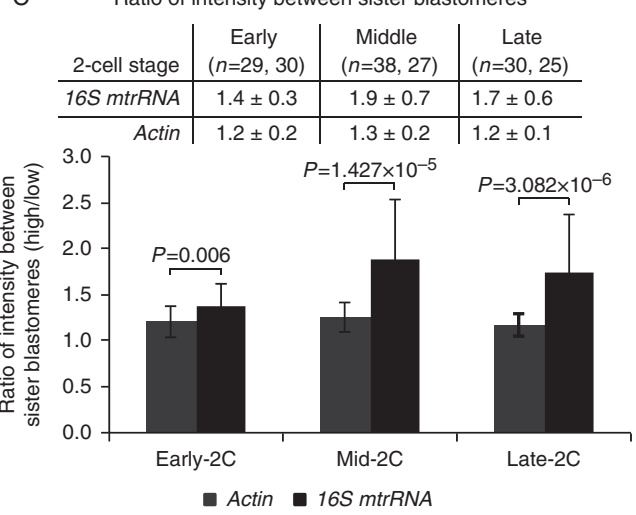

F Ratio of intensity between sister blastomeres

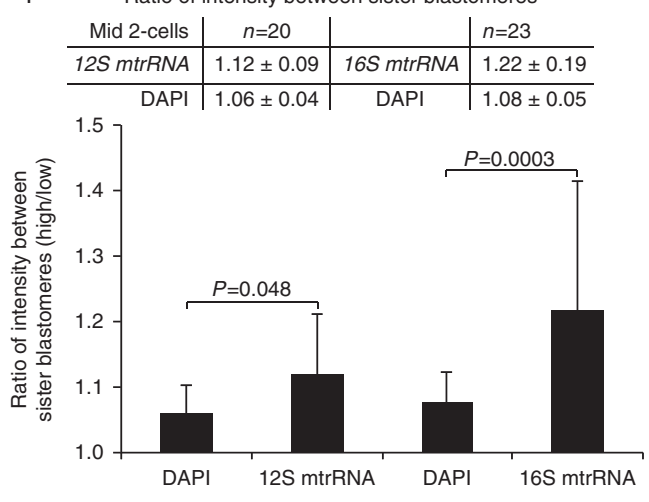

Figure 2 Distribution of mitochondrial ribosomal RNAs between mouse 2-cell sister blastomeres. (A) In situ hybridization of mouse 2-cell embryos. Mouse 2-cell embryos were separated into early, middle and late stages and hybridized with long cRNA probes for $12 S$ and $16 S$ mtrRNAs respectively. Actin was used as control. No signals above background were detected when embryos were hybridized with $165 \mathrm{mtr} R \mathrm{NA}$ sense probes. (B and C) Ratios of in situ hybridization signal intensities for Actin and mtrRNAs between 2-cell sister blastomeres ( $R=\mathrm{OD} 1 / \mathrm{OD} 2$, OD1 $>$ OD2). Embryonic stages, number of embryos measured and average ratios of signal intensities are shown in tables (upper panels). Bar graphs are displayed in lower panels. Significances of the differences between control and experimental groups were assessed using Mann-Whitney test for two independent samples. (D and E) Fluorescent in situ hybridization (FISH) of $12 S$ and $16 S$ mtrRNAs in mouse 2-cell embryos using Stellaris oligonucleotide probes (20-mers). Probes were tagged with Quasar 570 and Quasar 670 dyes for $12 S$ and $16 S$ mtrRNAs respectively. $16 S$ mtrRNA signal was pseudo-colored as green. (F) Quantification of FISH signals between 2-cell sister blastomeres. Comparing to DAPI staining, both mtrRNAs appeared higher in one blastomere than the other. Number of embryos measured and the average ratios of signal intensity are shown in table (upper panel). Bar graph is shown in lower panel. Significances of the differences between control and experimental groups were assessed using Mann-Whitney test. Error bars: S.D.

high variations and differences of mtrRNAs between sister blastomeres (Supplementary Figure S1G). We further quantified mtrRNA levels more directly using the fluorescent signals following FISH of mtrRNAs. Both
$12 \mathrm{~S}$ and $16 \mathrm{~S}$ mtrRNAs could be readily seen in the cytoplasm of blastomeres when 2-cells were hybridized with short fluorescent probes (Fig. 2D and E). Quantification of the fluorescent signals in paired sister 
blastomeres showed that 2-cell embryos contained differential levels of mtrRNAs in sister blastomeres (about $10-20 \%$ at the examined mid-2-cell stage), comparing with the equally distributed nuclear DAPI signals $(n=20,23 ; P=0.048,0.0003$ for $12 \mathrm{~S}$ and $16 \mathrm{~S}$ mtrRNAs respectively, Mann-Whitney test) (Fig. 2F).

It has been suggested that hormone treatment during superovulation or different mouse strains would influence the quality of embryos obtained, therefore casting environmental effects on development (Sanfins et al. 2003). To eliminate the possibility that these artificial treatments will deviate the distribution of mtrRNAs between 2-cell sister blastomeres, mid-2-cell embryos isolated from $\mathrm{CD} 1$ mice that were naturally mated or from $\mathrm{C} 57 \mathrm{BL} / 6$ mice that were superovulated was hybridized with DIG-labeled long cRNA probes. Comparing with the Actin controls that were processed at the same time, expression of $16 S \mathrm{mtrRNA}$ was found to be more differential between 2-cell sister blastomeres from these mice. The average ratios of ISH signal intensity for $16 S$ mtrRNA were significantly more variable $(1.4 \pm 0.3$ for CD1 mice, $n=23 ; 1.4 \pm 0.4$ for C57BL/6, $n=20$ ) comparing with those of Actin $(P<0.05, z$-test for two samples' means) (Supplementary Figure S1C, D, E and F). This suggested that the uneven distribution of $16 \mathrm{~S}$ mtrRNA between 2-cell sister blastomeres was not affected by hormone injection or different strains of mice. Taken together, these results indicated that mtrRNAs are differentially distributed between mouse 2-cell sister blastomeres.

To examine whether the asymmetric distribution of mtrRNAs would persist with further cell divisions, levels of mtrRNAs among blastomeres in 4-cell embryos were examined using ISH. To better visualize 4-cell sister blastomeres, they were first manually separated into single cells from each embryo (Fig. 3A). Groups of four sister blastomeres of the same embryo were then hybridized with the same long cRNA probes together. Signals of mtrRNAs in individual blastomeres were imaged and measured as described above. Relative signal ratios were compared among sister blastomeres when setting the blastomere with the highest intensity as 1. It was found that 4-cell sister blastomeres contained descending amounts of mtrRNAs, with two of them containing $\sim 20 \%$ less of mtrRNAs than the other two (Fig. 3B and C). The differences of $12 \mathrm{~S}$ and $16 \mathrm{~S}$ mtrRNAs among sister blastomeres were significantly varied $(P<0.05, n=22$ and 16 respectively), comparing with those of Actin $(P=0.438, n=15$, one-way ANOVA, $\alpha=0.05$ ) (Supplementary Tables S4 and S5). The high variations and differences of mtrRNA levels among 4-cell sister blastomeres were also seen using single cell quantitative RT-PCR (Supplementary Figure S1H). These results suggested that the asymmetric distribution of mtrRNAs could be propagated with further cell divisions. Recent studies showed that mouse 4-cell blastomeres contain differentially expressed epigenetic
A
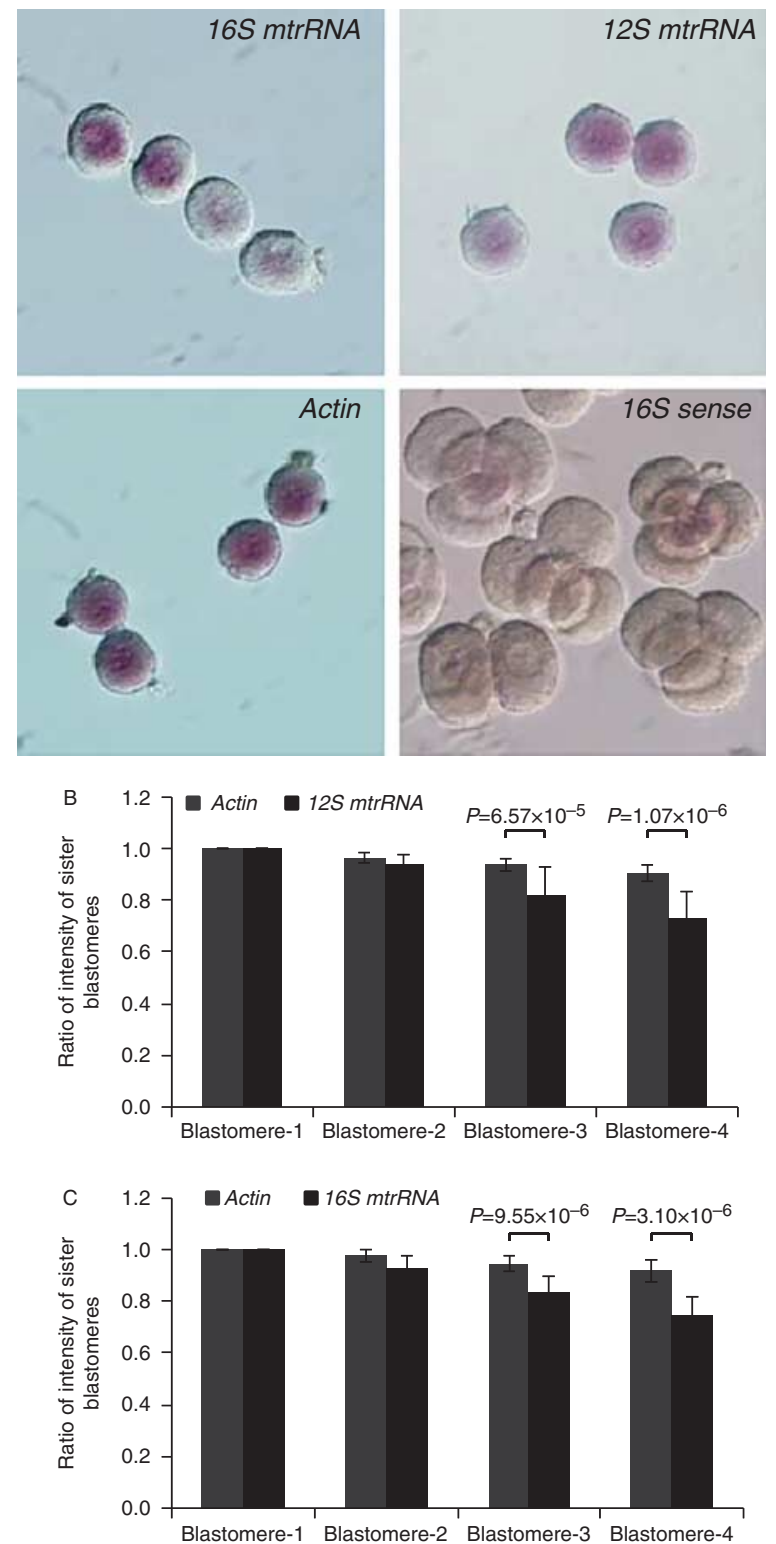

Figure 3 Distribution of mtrRNAs among sister blastomeres of mouse 4-cell embryos. (A) In situ hybridization of mouse 4-cell embryos. Sister blastomeres of 4-cell embryos were separated manually by micromanipulation before being hybridized together with indicated probes. Shown are representative light micrographs. Images of in situ hybridization with $16 S \mathrm{mtrRNA}$ sense probe from a separate experiment was included as control. (B) Ratios of in situ hybridization signal intensities for Actin and $12 S \mathrm{mtrRNA}$ among 4-cell sister

blastomeres. Comparing to the sister blastomere with the highest signal intensity (set as 1), ratios of Actin and $12 S$ mtrRNA signals for the two lowest blastomeres are $0.93 \pm 0.02,0.90 \pm 0.03(n=16)$ and $0.82 \pm$ $0.11,0.73 \pm 0.1(n=22)$, respectively. (C) Ratios of in situ hybridization signal intensities for Actin and 165 mtrRNA among 4-cell sister blastomeres. Comparing with the sister blastomere with the highest signal intensity (set as 1), ratios of Actin and $165 \mathrm{mtrRNA}$ signals for the two lowest blastomeres are $0.95 \pm 0.03,0.92 \pm 0.04(n=15)$ and $0.83 \pm 0.07,0.75 \pm 0.07(n=16)$ respectively. Significances of the differences between control and experimental groups were assessed using Mann-Whitney test (see also Supplementary Tables S4 and S5). Error bars: S.D. 
modifiers that influence the destination of respective progeny cells in blastocyst (Torres-Padilla et al. 2007, Burton et al. 2013). Although it is not clear at this stage whether the differentially distributed mtrRNAs in mouse 2-cell embryos are related to the differential gene expression at later stages, the results nevertheless suggest that molecular asymmetry occurs in mouse 2-cell embryos, earlier than previously found embryonic stage.

\section{Mouse 2-cell embryos contain cytoplasmic 165 mtrRNA outside of mitochondria}

Since mtrRNAs are essential components of mitochondrial ribosomes, whether the differential localization of mtrRNAs reflects differences in mitochondria distribution or mitochondrial activity between 2-cell sister blastomeres was examined next. Whole-mount immunofluorescent staining of embryos with MitoTracker Red showed overall even distribution of mitochondria in the cytoplasm of 2-cell blastomeres. Quantification of MitoTracker Red signals in paired sister blastomeres of 2-cell embryos from confocal micrographs gave rise to the ratio of $1.1 \pm 0.08$ between sister blastomeres, similar to that of nuclear DAPI staining ( $R=1.1 \pm 0.07, n=30)$, indicating that 2-cell sister blastomeres contain similar amounts of mitochondria (Fig. 4A and B). Interestingly, in contrary to apparent higher levels of mtrRNAs in inner cells of morula and blastocyst (Fig. 1A and B), fluorescent signals of MitoTracker Red in the inner cells appeared weaker than those of outer cells (Supplementary Figure S2A, see section on supplementary data given at the end of this article), suggesting a probable higher mitochondria contents in cells at the outer layer of morula and blastocysts. Next, the membrane potential of mitochondria was examined using TMRM staining of live 2-cell embryos. Comparing to embryos treated with FCCP (the membrane potential un-coupler), live embryos were readily stained by the dye (Fig. 4C). Little difference in the intensity of TMRM staining was found between 2 -cell sister blastomeres $(R=1.1 \pm 0.08$, $n=40$ ), similar to that of nuclear DNA staining $(R=1.08 \pm 0.12$, Fig. 4D), suggesting that 2-cell blastomeres also have similar mitochondrial activity. Thus, neither the quantity nor the activity of mitochondria is differentially distributed between sister blastomeres of 2-cell embryos.

One possibility for un-evenly distributed mtrRNAs seen in 2-cell embryos is the incomplete hybridization of long anti-sense cRNA probes due to their difficulty to penetrate mitochondrial membranes, as previously suggested (Ninomiya \& Ichinose 2007). To test this, the expression of two mitochondria encoded genes $(\mathrm{Nd} 2$ and $C y t b)$ and a nuclear gene $(C y c 1)$ was analyzed using respective long CRNA probes. Quantitative RT-PCR first showed that both $N d 2$ and $C y t b$ were expressed at levels higher than that of $16 S \mathrm{mtrRNA}$ at most pre-implantation
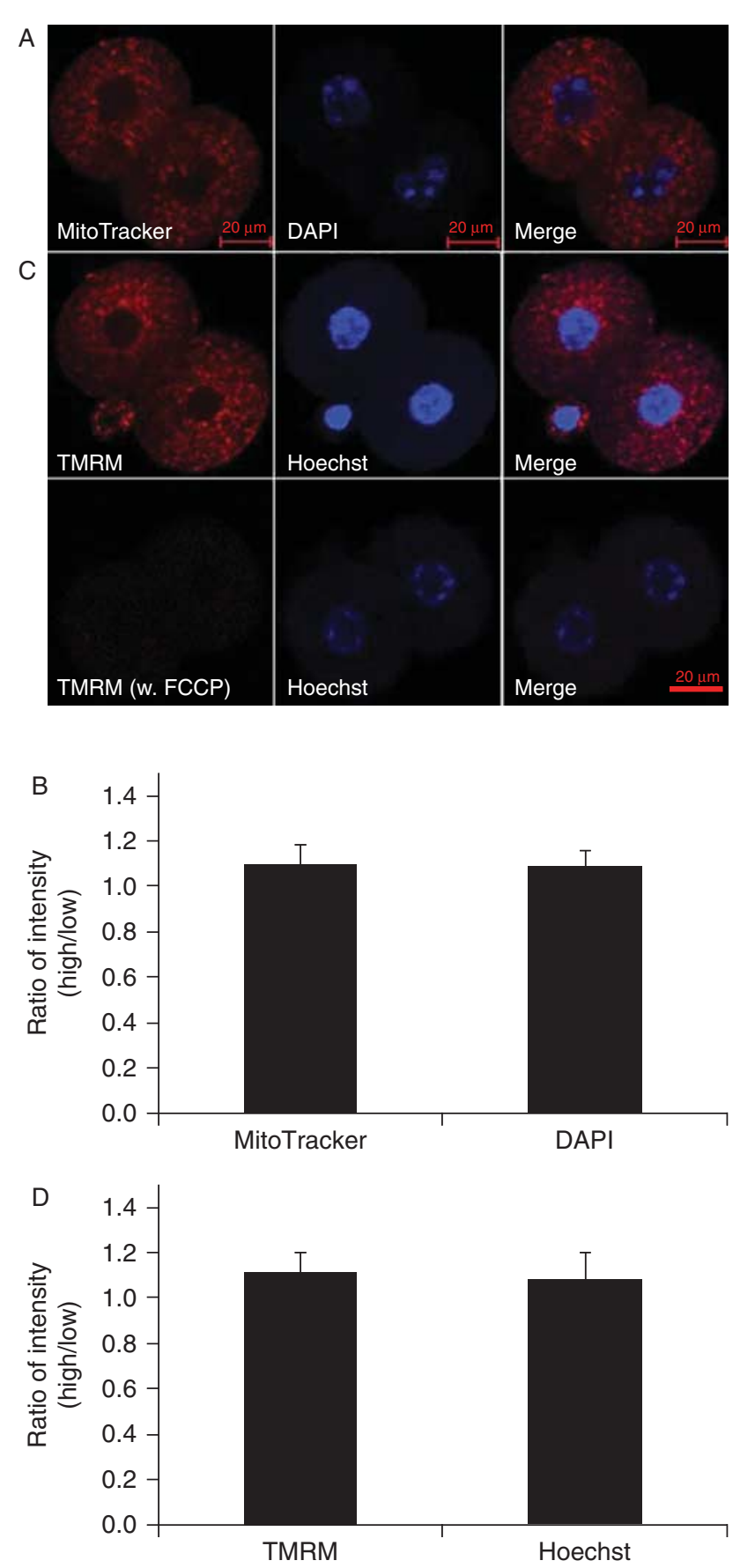

Figure 4 Mouse 2-cell embryos contain overall equal amount and activity of mitochondria. (A) MitoTracker Red staining of mouse 2-cell embryos. Nuclei were stained with DAPI. Shown are representative confocal images. (B) Ratio of signal intensities of MitoTracker Red between 2-cell sister blastomeres. Similar ratios of mitochondria $(R=1.09 \pm 0.09)$ and DAPI $(R=1.09 \pm 0.07, n=30)$ indicate equal amounts of mitochondria between sister blastomeres. (C) TMRM staining of live 2-cell embryos. As control, FCCP was used to un-couple mitochondrial membrane potential before TMRM staining (lower panels). Nuclei were stained with Hoechst 33342. Shown are representative confocal images. (D) Ratio of TMRM signal intensities between 2-cell blastomeres. Similar ratios of TMRM $(R=1.12 \pm 0.08)$ and Hoechst $(R=1.08 \pm 0.12, n=40)$ indicate the equal mitochondrial activity between sister blastomeres. 
embryonic stages, whereas $C y_{c} 1$ was expressed at much lower level (Fig. 5A). However, both mRNAs of $\mathrm{Nd} 2$ and $C y t b$ that are known to be inside of mitochondria were not detected by ISH, whereas Cyc1 mRNA was readily seen in morula and blastocysts using the same method (Fig. 5B). This suggested that long cRNA probes was able to detect relatively small amount of mRNAs in the cytoplasm of cells during ISH, but may indeed be difficult to localize RNA species within mitochondria. It also suggested that the differentially distributed mtrRNAs detected by long cRNA probes were probably localized outside of the organelle. Since small
A

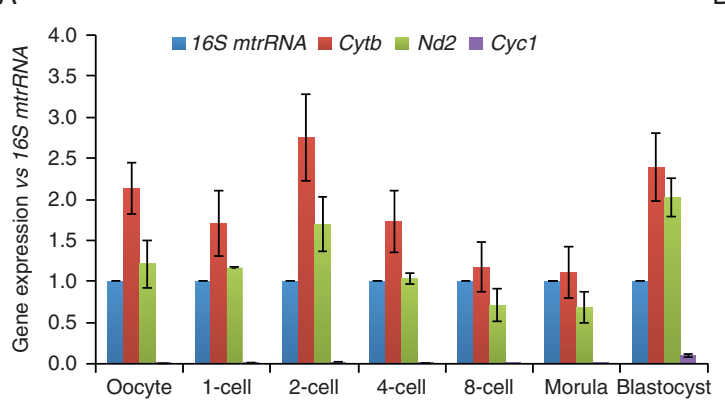

B

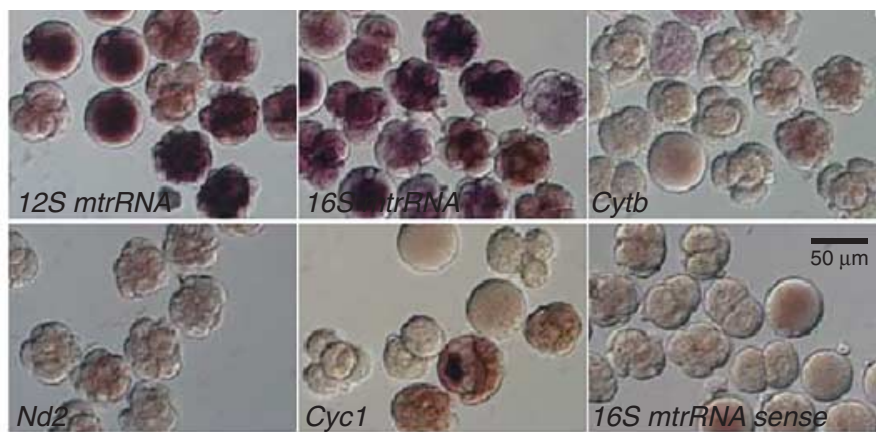

C
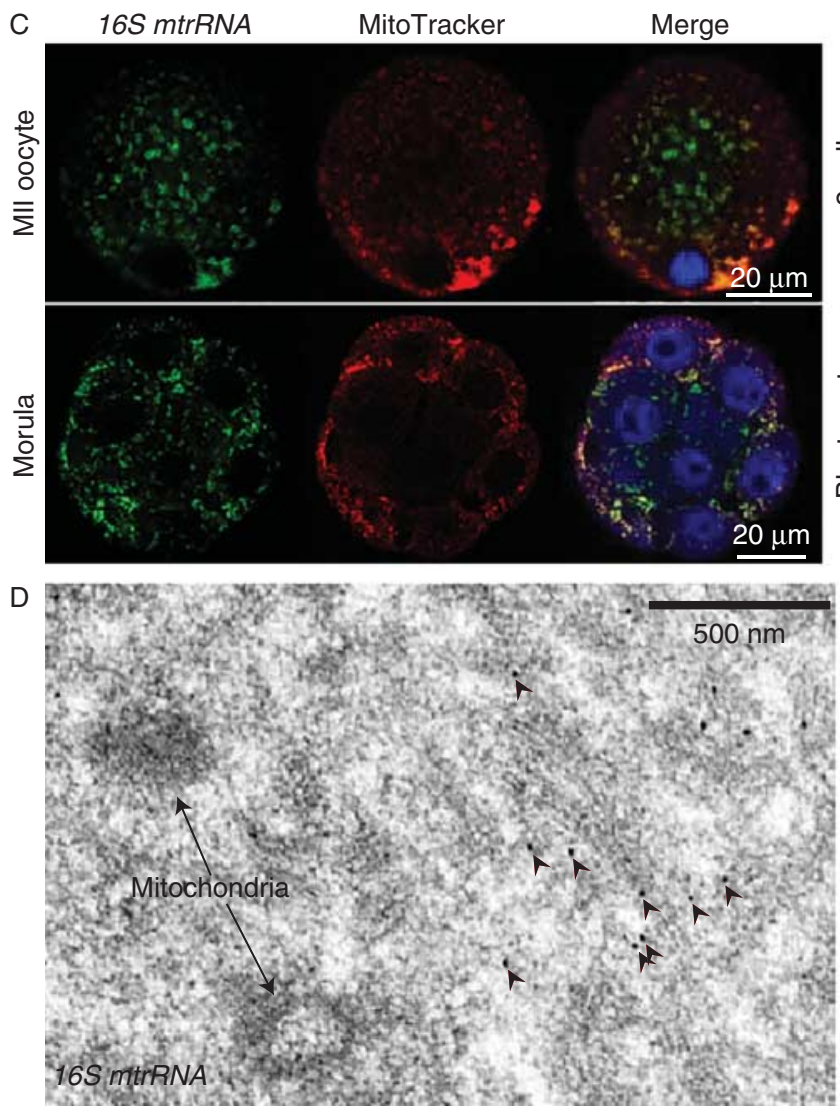
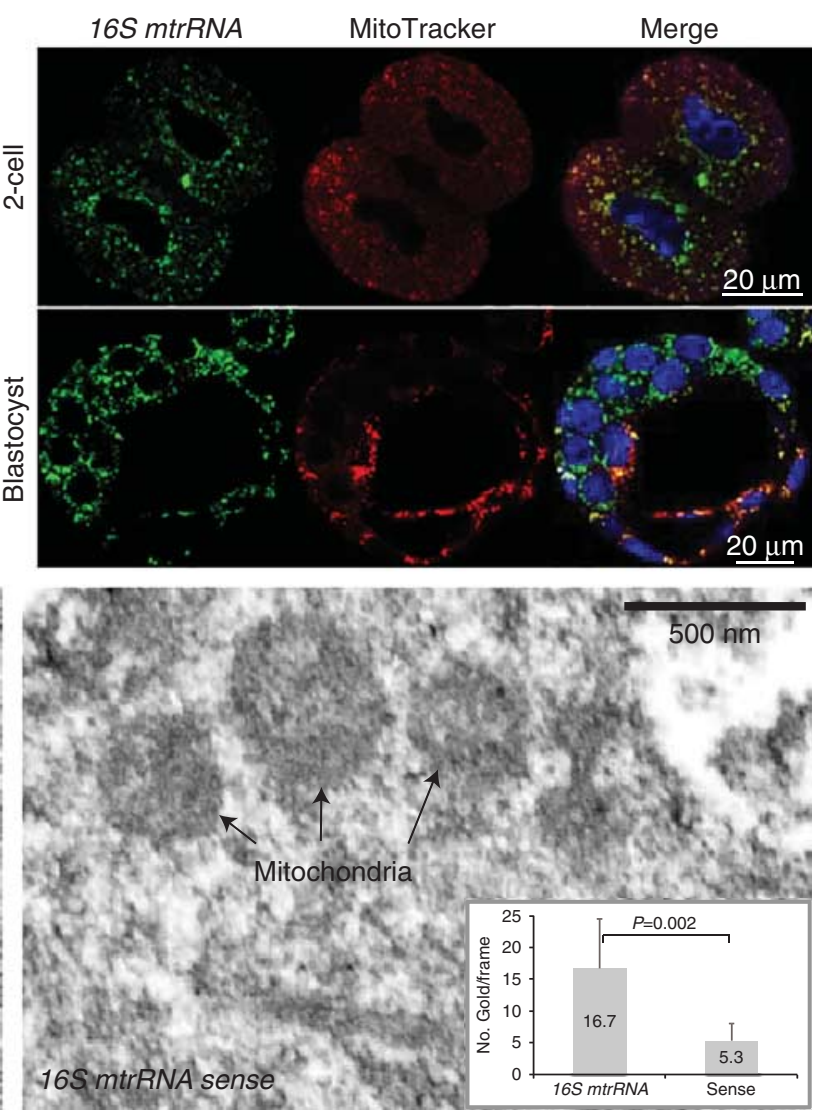

Figure 5 Distribution of cytoplasmic 16S mtrRNA outside of mitochondria in mouse 2-cell embryos. (A) Quantitative RT-PCR of mitochondrial genes. Shown are average expression levels relative to that of $165 \mathrm{mtrRNA}$ from three independent experiments. (B) In situ hybridization of pre-implantation mouse embryos. Collected embryos (from 1-cell to blastocyst) were hybridized with long cRNA probes for indicated genes. Signals for mitochondria encoded $C y t b$ and $\mathrm{Nd} 2$ were not detectable in cytoplasm despite their higher levels of expression (A), whereas nuclear encoded $C y c 1$ could be seen at morula and blastocyst stages. Anti-sense and sense probes for mtrRNAs were used as controls. (C) Co-staining of 16S mtrRNA and mitochondria. Signals of $16 \mathrm{~S}$ mtrRNA (detected by fluorescent oligonucleotide probes) were found to partially co-localize with mitochondria (detected by MitoTracker Red) from MII oocytes to blastocysts. Inner cells of morula and blastocysts contain higher levels of 16S mtrRNA than MitoTracker Red signals. (D) In situ hybridization of 165 mtrRNA at electron microscopic level (ISH-EM). 16S mtrRNA (arrow heads) was detected in the cytoplasm outside of mitochondria (arrows), as revealed by anti-DIG-colloidal gold secondary antibodies. Sense probe was used as negative control (right panel). Average numbers of gold particles per frame were calculated from ten frames of electron micrographs in each group (Inset, $n=10$ ). Significance of the difference was assessed using Mann-Whitney test. Error bars: s.D. 
oligonucleotide probes could better penetrate mitochondria, FISH signals of mtrRNAs may represent both cytoplasmic and mitochondrial fractions of mtrRNAs. Using fluorescently tagged oligonucleotide FISH probes, together with MitoTracker Red, the subcellular localization of $16 \mathrm{~S}$ mtrRNA in mouse embryos was further examined. In control experiments, fluorescent signals of $16 \mathrm{~S}$ mtrRNA were found to completely overlap with that of MitoTracker Red in mouse embryonic fibroblast (MEF) and 293T cells (Supplementary Figure S2B). However, in mouse MII oocytes and pre-implantation embryos, signals of $16 \mathrm{~S}$ mtrRNA were found to only partially overlap with MitoTracker Red staining, suggesting that $16 \mathrm{~S}$ mtrRNA was also present in the cytoplasm outside of mitochondria in mouse female germ cells and embryos (Fig. 5C). Green fluorescent signals of $16 \mathrm{~S}$ mtrRNA that did not overlap with MitoTracker Red were found in the cytoplasm of MII oocytes toward animal pole and more concentrated in inner cells in morula and blastocysts, suggesting that $16 \mathrm{~S}$ mtrRNA at these regions was likely outside of mitochondria, consistent with ISH results using long CRNA probes (Fig. 1).

Mitochondrial rRNAs have been found outside of mitochondria in early embryos of Drosophila, Sea urchin and Xenopus (Kobayashi et al. 1993, 1998, Ogawa et al. 1999). In mouse, it has been suggested that MII oocytes also contain mtrRNAs in the cytoplasm (Ninomiya \& Ichinose 2007). To further determine the sub-cellular localizations of $16 \mathrm{~S}$ mtrRNA in relation to mitochondria, 2-cell embryos were examined at electron microscopic level following ISH. In control experiment, transmission electron microscopy of thin sections $(100 \mathrm{~nm})$ of 2-cell embryos showed that mitochondria appeared to be spherical in shape with clear cristae (Supplementary Figure S2C). Following ISH, mitochondria were less well maintained and the internal cristae of mitochondria were sometimes lost due to repeated sample treatments. However, the overall morphology of mitochondria remained identifiable (Supplementary Figure S2D). Using anti-DIG antibody conjugated with 12-nm colloidal gold particles, it was found that $16 \mathrm{~S}$ mtrRNA was distributed in the cytoplasm of 2-cell blastomeres outside of mitochondria (Fig. 5D). The average number of gold particles on thin sections of 2-cell embryos was significantly higher when anti-sense probes of $16 \mathrm{~S}$ mtrRNA were used $(16.7 \pm 7.8)$, comparing with that of sense control $(5.3 \pm 2.7, P=0.002, n=10$, Mann-Whitney test) (Fig. 5D, inset). Collectively, these results indicate that mouse 2 -cell blastomeres contain $16 \mathrm{~S}$ mtrRNA in the cytoplasm outside of mitochondria.

\section{5 mtrRNA in 2-cell embryos regulates cell allocations in blastocyst}

First found in Drosophila, $16 \mathrm{~S}$ mtrRNA was shown to drive the germ cell formation during early embryogenesis (lida \& Kobayashi 1998). To find out whether the differentially expressed $16 \mathrm{~S}$ mtrRNA in mouse 2-cell embryos could have functional roles in cell-lineage allocations during pre-implantation embryogenesis, levels of $16 \mathrm{~S}$ mtrRNA were altered in one sister blastomere of 2-cell embryos using microinjection of either sense or antisense RNAs. In vitro transcribed Egfp mRNA was used as control or co-injected into the same blastomere in order to trace the fate of its descendent into blastocysts (Fig. 6A). Injected embryos were developed till blastocyst stage in vitro and scanned at $2-\mu \mathrm{m}$ interval using confocal microscopy. Confocal sections of each individual blastocyst were compiled into single 3-D images, from which the number and locations of cells were analyzed (Supplementary Movies 1 and 2, see section on supplementary data given at the end of this article). In the three dimensional space, cells that are located on the outer-most layer on either axis of the three dimensions ( $\mathrm{X}, \mathrm{Y}$ and $\mathrm{Z}$ ) were considered as the TE cells, whereas cells that are surrounded by others on all three axes were counted as the ICM cells (Fig. 6B). Cell positions were determined by the nuclear DAPI staining and green fluorescence was used to identify the EGFP-labeled $\left(\mathrm{EGFP}^{+}\right)$cells. For the ease of analyses of 3D cell allocations, embryos containing 40-45 cells (46-48 $\mathrm{h}$ in vitro culture following microinjection), corresponding to early blastocysts were used in most cases. At this stage, ICM and TE cells are segregated, whereas epiblast and primitive endoderm cells of the ICM are yet to be differentiated. To confirm the cell positioning method, embryos were co-stained with cellcell junctional E-Cadherin or plasma membrane marker TRITC-Phalloidin in some cases so that cell boundaries were clearly defined. It was found that cells in both ICM and TE could be similarly positioned with or without cell membrane labeling (Supplementary Figure S3). Thus for most of the cell allocation analyses, embryos without cell membrane labeling were used. Total numbers of $\mathrm{EGFP}^{+}$cells that were allocated into either ICM or TE were then counted in order to assess the effects of changing $16 \mathrm{~S}$ mtrRNA levels on cell lineage allocations.

Quantitative RT-PCR showed that anti-sense mtrRNA decreased 16S mtrRNA level in injected blastomeres, while sense RNA increased it by about 60\% (Supplementary Figure S4A, see section on supplementary data given at the end of this article). The decrease of $16 \mathrm{~S}$ mtrRNA following anti-sense microinjection was also supported by the decreased fluorescent signals in injected blastomeres when compared to the noninjected ones using FISH (Supplementary Figure S4B). Examination of Egfp mRNA-injected embryos first showed that EGFP $^{+}$cells derived from microinjected blastomere occupied blastocyst in both ICM and TE without affecting the overall development of embryos in vitro. All groups of blastocysts contained similar numbers of cells when cultured for the same length of time in vitro (Supplementary Table S6). There were about $34 \%$ of $\mathrm{EGFP}^{+}$cells in ICM and $66 \%$ of $\mathrm{EGFP}^{+}$ cells in TE in Egfp-injected embryos (Fig. 6F). Comparing 
with the Egfp-injected control, increasing 16S mtrRNA (sense injection) increased the number of EGFP ${ }^{+}$ descendent in ICM (to about $49 \%)(P=0.005, n=18)$, while reduced $16 \mathrm{~S}$ mtrRNA level (anti-sense injection) caused an increase in the number of $\mathrm{EGFP}^{+}$cells in TE (to about $78 \%)(P=0.0001, n=19$, Mann-Whitney test) (Fig. 6F and Supplementary Table S6). These changes of cell allocations of $\mathrm{EGFP}^{+}$cells following microinjection appeared statistically significant when they were compared together across different conditions $(P<0.001$, one-way ANOVA, $\alpha=0.05$ ) (Supplementary Table S7).

The cell allocation effects of altered 16S mtrRNA levels were further examined by cell lineage specific gene expression. Using antibodies against cell-lineage markers NANOG (pluripotency marker) and CDX2 (TE marker), in vitro cultured blastocysts were immunostained following microinjection. It was found that patterns of marker gene expression in either ICM or TE cell lineages were not changed with respective allocations in blastocyst following either sense or anti-sense
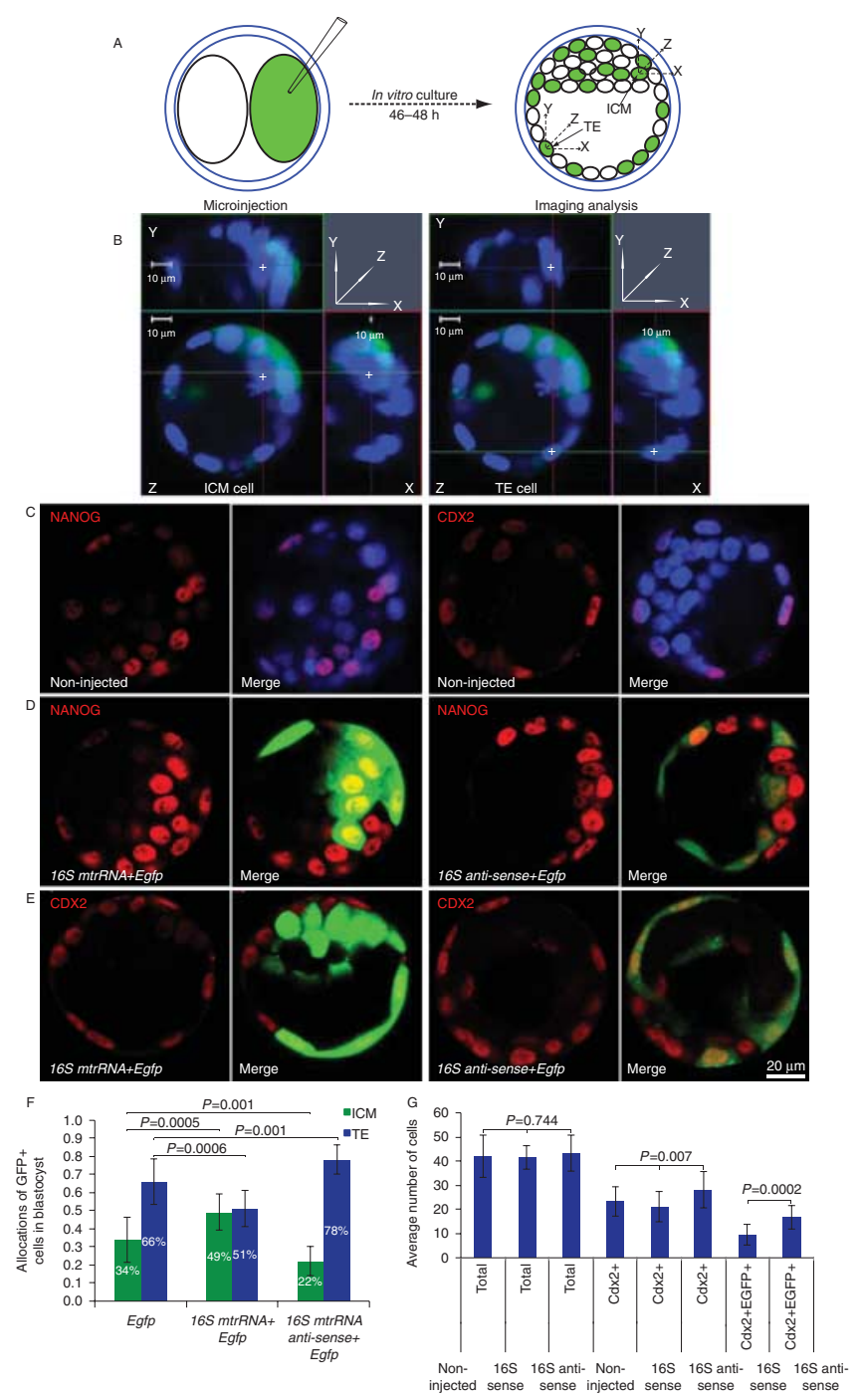

injection, comparing to the non-injected control group (Fig. 6C, D and E). NANOG was primarily found in ICM cells with occasional staining in TE cells, whereas CDX2 was exclusively localized in TE cells at the outer layer in all groups. Since NANOG is a pluripotency marker but not a strict ICM specific marker at the early blastocyst stage and CDX2 is an exclusive TE lineage marker, changes of $\mathrm{CDX} 2$ expressing $\left(\mathrm{CDX} 2^{+}\right)$cells in microinjected embryos were further analyzed. It was found that, while overall development of the embryos remained unaltered (embryos contained similar number of cells during similar period of culturing time in all groups), $\mathrm{EGFP}^{+}$cells contained higher fraction of $\mathrm{CDX}^{+}$cells $\left(16.7 \pm 5.0,69.5 \pm 13.3 \%\right.$ of total $\mathrm{EGFP}^{+}$ cells) when $16 S$ anti-sense was injected, comparing with sense injected embryos $(9.4 \pm 4.2,42.6 \pm 16 \%$ of total EGFP $^{+}$cells, $P=0.0002, n=18-19$, Mann-Whitney test) (Fig. 6G, Supplementary Tables S8 and S9). These results suggested that changes of $16 \mathrm{~S}$ mtrRNA did not change the marker gene expression prior to cell lineage allocations and levels of $16 \mathrm{~S}$ mtrRNA in 2-cell blastomeres could deviate the segregation of ICM and TE cell lineages in blastocyst. Neither anti-sense nor sense $16 \mathrm{~S}$ mtrRNA brought changes to mitochondrial

Figure 6 Influences of 16S mtrRNA in 2-cell blastomeres on cell allocations in mouse blastocyst. (A) Schematic drawing of 2-cell embryo microinjection. In vitro transcribed Egfp mRNA was injected into one blastomere at random, either singly or together with $16 \mathrm{~S}$ mtrRNA. Embryos were cultured in vitro for $46-48 \mathrm{~h}$ till blastocyst stage and $\mathrm{EGFP}^{+}$descendent cells were traced for their locations in either inner cell mass (ICM) or trophectoderm (TE) within blastocyst. (B) Representative confocal images showing locations of EGFP ${ }^{+}$ICM and TE cells. Blastocysts cultured in vitro following microinjection were scanned under a confocal microscope at $2-\mu \mathrm{m}$ interval. EGFP ${ }^{+}$ descendent cells were traced for their locations as marked by DAPI staining using 3-D compository images. Cells that are surrounded by other cells along three axes ( $\mathrm{X}, \mathrm{Y}$ and $\mathrm{Z}$ ) are denoted as ICM cells (left panel) and cells that are located on the outside along either one of the three axes are denoted as TE cells (right panel). Shown are center sections of a scanned blastocyst with indicated ICM and TE cells on three dimensions. (C, D and E) Immunostaining of NANOG and CDX2 in mouse blastocysts with or without microinjection. Embryos were cultured in vitro from 2-cell stage, when one blastomere was microinjected with indicated RNA species. Similar to the control (C), NANOG was found to primarily express in ICM cells, with occasional staining in TE cells (D), whereas CDX2 was found to express exclusively in TE cells in all groups (E). Cell nuclei were stained with DAPI. Representative confocal images of immunostaining of NANOG or CDX2 are shown on the left and merged images with either DAPI or EGFP signals are shown on the right in each panel. (F) $16 \mathrm{~S}$ mtrRNA influences cell allocations in blastocyst. Comparing to Egfp mRNA injected controls, $165 \mathrm{mtrRNA}$ sense or anti-sense increased EGFP ${ }^{+}$ cells' occupation in either ICM or TE, respectively ( $n=18-19$; see also Supplementary Tables S6 and S7). (G) While total number of cells were not changed in blastocysts with or without microinjection, $165 \mathrm{mtrRNA}$ anti-sense increased $\mathrm{CDX}^{+}$cells in $\mathrm{EGFP}^{+}$fraction $(n=18-20$; see also Supplementary Tables S8). Pair-wised comparison was assessed for significance using Mann-Whitney test, while significance of differences among three groups were assessed using one-way ANOVA and post-hoc Turkey analyses (see also Supplementary Table S9). 
activity between 2-cell blastomeres (Supplementary Figure S4C and D), suggesting that the extra-mitochondrial fraction of the mtrRNA may be accountable for the cell lineage allocation effects.

Since bi-laterally symmetric mouse blastocysts can be divided into embryonic half (containing ICM and polar TE) and abembryonic half (containing mural TE) along the $\mathrm{A}-\mathrm{V}$ axis, cell allocation effects of $16 \mathrm{~S}$ mtrRNA along the A-V axis following microinjection was further analyzed. Based on the distribution of $\mathrm{EGFP}^{+}$descendent in either embryonic or abembryonic allocations in blastocysts, embryos were separated into three different categories (Fig. 7A and B). Among 101 Egfp-injected embryos, $31.7 \%$ of embryos contained EGFP ${ }^{+}$progenies only at embryonic side (pattern 1, embryonic) and $25.7 \%$ of embryos contained EGFP $^{+}$cells only at abembryonic side (pattern 3, abembryonic). The remaining $42.6 \%$ of embryos contained $\mathrm{EGFP}^{+}$cells in both cell allocations (pattern 2, mixed). However, when single 2-cell sister blastomeres were co-injected with anti-sense $16 \mathrm{~S} m \mathrm{mtRNA}$ and Egfp mRNA, blastocysts containing $\mathrm{EGFP}^{+}$progenies in embryonic half reduced from $31.7 \%$ to $15.3 \%$, whereas blastocysts containing $\mathrm{EGFP}^{+}$cells in the mixed cell allocations increased to $68.1 \% \quad(n=144)$. Including the remaining $16.7 \%$ embryos with $\mathrm{EGFP}^{+}$cells located only at abembryonic half, a total of $84.8 \%$ embryos contained progenies from injected blastomeres at abembryonic allocations (comparing to the total of $68.3 \%$ in the control group). The dramatic increase of embryos with mixed cell allocations is probably caused by the randomness at choosing the 2-cell sister blastomeres for microinjection. In contrast, when in vitro transcribed 16S mtrRNA was co-injected with Egfp mRNA into single 2-cell blastomeres, blastocysts containing $\mathrm{EGFP}^{+}$descendent allocated to embryonic cell-lineage increased to $46.5 \%$, whereas blastocysts with $\mathrm{EGFP}^{+}$abembryonic cells decreased to $12.6 \% \quad(n=127)$ (Fig. 7C). Statistical analysis suggested that these changes of embryos in different patterns were significantly relevant to the microinjection of either sense or anti-sense $16 \mathrm{~S}$ mtrRNAs among experimental groups $\left(\chi^{2}=39.18, P<0.001, \chi^{2}\right.$ test, Supplementary Table S10). Taken together, these results suggested that the increase of $16 \mathrm{~S}$ mtrRNA in 2-cell blastomeres facilitates their progeny cells to allocate into pluripotent embryonic cell lineage, whereas reduction of $16 \mathrm{~S}$ mtrRNA deviates the descendent into abembryonic allocation.

\section{Discussion}

It has been reported that the total number of mitochondria and the mitochondrial DNA remain largely unchanged in pre-implantation mouse embryos (Piko \& Taylor 1987). Since embryonic mitochondria are mostly inherited from the maternal source (Cree et al. 2008, Mishra \& Chan 2014), the number of mitochondria in

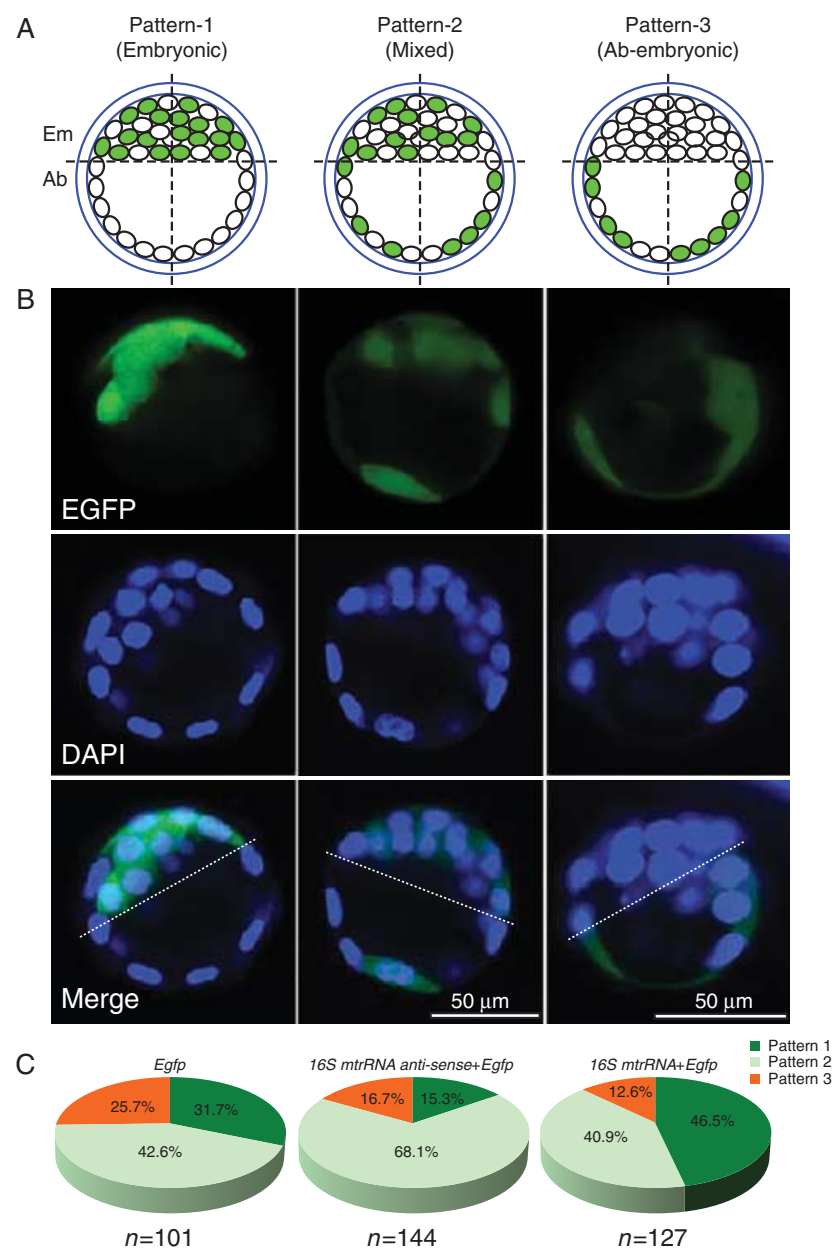

Figure 7 Influences of $16 \mathrm{~S}$ mtrRNA in 2-cell blastomeres on cell lineages of blastocyst. (A) Schematic drawing of categorization of blastocysts containing differentially distributed $\mathrm{EGFP}^{+}$progeny cells. Pattern 1: $\mathrm{EGFP}^{+}$descendent found only at embryonic side (left panel); pattern 2: $\mathrm{EGFP}^{+}$descendent found at both embryonic and abembryonic sides (mixed, middle panel); pattern 3: EGFP ${ }^{+}$descendent found only at abembryonic side (right panel). (B) Representative confocal micrographs of blastocysts developed from microinjected 2-cell embryos, corresponding to three patterns depicted in (A). Cell nuclei were stained with DAPI. Dotted lines show the embryonicabembryonic boundary. (C) Changes of embryonic patterning in blastocyst following 2-cell blastomere microinjection with $16 \mathrm{~S}$ mtrRNA or anti-sense RNAs. One sister blastomere of 2-cell embryo was microinjected with indicated RNAs. Comparing to the Egfp-injected control, 16S mtrRNA anti-sense decreased descendent allocation in embryonic cell lineage (pattern 1, green) and increased their mixed cell allocations (pattern 2, light green), while 16S mtrRNA increased $\mathrm{EGFP}^{+}$cells in embryonic cell lineage and decreased their abembryonic allocation (pattern 3, orange). Numbers of embryos analyzed are indicated below pie charts.

embryonic cells gradually decreases with cell division until post-implantation stage when cell proliferation accelerates. However, mtrRNAs are actively transcribed during pre-implantation development (Piko \& Chase 1973, Piko \& Taylor 1987). Consistent with this, quantitative RT-PCR showed that the level of $16 \mathrm{~S}$ 
$m$ trRNA is maintained constant from MII oocytes to 2-cell embryos and increased dramatically after 4-cell stage. This suggests that mitochondrial genome is probably expressed continuously in the absence of DNA replication, separating its gene expression regulation away from that of nuclear genome (Lee et al. 2014).

In unfertilized eggs, mtrRNAs appeared to localize more towards the animal pole where the first polar body emits. Uneven distribution of mtrRNAs was also observed throughout early embryos at different stages from 2-cell and 4-cell (higher in half of the blastomeres) to morula and blastocyst (higher in inner cells). It is not clear at this stage whether the maternal polarity is related to the molecular asymmetry observed in early embryos. Several studies have shown that LEPTIN and STAT3 are asymmetrically localized in unfertilized eggs and early embryos in mouse, accumulating at the animal pole toward the first polar body (Antczak \& Van Blerkom 1997, Schulz \& Roberts 2011). This is similar to that of mtrRNAs in unfertilized eggs (Fig. 1A and B). A large multi-protein complex (subcortical maternal complex (SCMC)) was also found to localize in the subcortical regions of mouse eggs and early embryos. Genetic depletion of the SCMC components in mouse caused developmental retardation at 2-cell stage (Li et al. 2008). During compaction, these polarized factors accumulate differentially in outer cells vs inner cells, generating polarity gradient within the embryo that may be linked to the cell-lineage determination and axis formation in blastocyst. However, none of them was found to differentiate in-between sister blastomeres following fertilization when embryogenesis starts. Recent research suggested that differentially localized epigenetic factors appearing at very early stage (4-cell stage) might regulate cell lineage determination in blastocysts (Torres-Padilla et al. 2007, Plachta et al. 2011, Burton et al. 2013). However, how these early molecular asymmetries are generated and whether there are even earlier cell fate determinants in mouse pre-implantation embryos remain elusive. Results presented here indicate that mouse 2-cell sister blastomeres contain quantitatively different mtrRNAs, suggesting that the molecular asymmetry may occur at the very beginning of mouse embryogenesis. Whether the differential mtrRNAs in 2-cell blastomeres are functionally related to the molecular asymmetries that occur during the development of early embryos requires further exploration.

Although the origin of differentially distributed mtrRNAs remains to be determined, their differences between sister blastomeres appear to be more evident at late 2-cell stage. One possibility is that the partitioned maternal mtrRNAs are differentially retained or degraded with the development of embryos. The transcription activity of mitochondrial genome could also differ between sister blastomeres. Alternatively, since the differential distribution of mtrRNAs were detected mainly outside of the organelle, mitochondria in sister blastomeres could export differential amount of mtrRNAs into the cytoplasm. It will be interesting to find out whether the post-transcriptional modifications of RNAs and mitochondrial activities contribute to the differential distribution of mitochondrial small and large ribosomal RNAs between 2-cell sister blastomeres. Although it is known that embryonic mitochondria are mainly inherited from the maternal source, it remains possible that sperm entry or the paternal cellular components could contribute to the regulation of embryogenesis beyond fertilization and activation of zygote. Previous sperm labeling and tracing experiment in mouse showed that sperm tail enters the egg during fertilization and remains in one of the 2-cell blastomeres (Jefferson \& Williams 2012). The experiment showed that sperm tail remained largely intact at 2-cell stage. Given that sperm mid-piece contains mitochondrial sheath with rather compacted configuration, this is different from what's seen with unevenly distributed mtrRNAs, which have diffused localization patterns. Research in Drosophila showed that sperm mitochondria are destroyed through autophagic and endocytic effects following fertilization (Politi et al. 2014). Whether paternal mitochondria are also eliminated in the same manner in mouse remains to be determined. Previous studies suggested that the mode of the first cell divisions is relevant to the progenies' cell lineage allocations in blastocysts (Piotrowska et al. 2001, Piotrowska-Nitsche \& Zernicka-Goetz 2005). Sequential meridionalequitorial divisions of 2-cell blastomeres showed more predictable fate of the descendent in the blastocyst (the blastomere that divide meridionally first tend to allocate into the embryonic hemisphere) than 2-cell blastomeres that are divided in the same directions (meridionalmeridional or equatorial-equatorial divisions). Perhaps the non-uniform dividing orientations could help to generate molecular gradients among progenies which are necessary preludes for cells' further differentiation. In this regard, it will be interesting to find out whether the levels of mtrRNAs are relevant to the regulation of the first cell divisions and cell lineage allocations in early embryos.

How are mitochondria encoded mtrRNAs localized in the cytoplasm outside of the organelle? Although mtrRNAs outside of mitochondria have been found in various species, including Drosophila, Sea urchin, Xenopus and mouse (Kobayashi et al. 1993, 1998, Ogawa et al. 1999, Ninomiya \& Ichinose 2007), it is not clear what molecular mechanisms that govern their cytoplasmic distribution. It was suggested that various RNA species encoded by mitochondrial genome could be exported into cytoplasm of human cells via unknown mechanisms (Attardi \& Attardi 1968, Maniataki \& Mourelatos 2005). One intriguing phenomenon is that neither the number nor the membrane potential of mitochondria has apparent differences between 2-cell 
sister blastomeres. It is known that mouse pre-implantation embryos utilize pyruvate and lactate instead of glucose to meet their energy and metabolic requirements and maintain relatively dormant mitochondrial oxidative phosphorylation activity until blastocyst stage (Krisher \& Prather 2012). Interestingly, inner cells of morula and blastocyst appear to contain higher levels of mtrRNAs when long CRNA probes were used for hybridization, whereas outer cells showed higher contents of mitochondria as indicated by TMRM staining (Fig. 1A and B, Supplementary Figure S2A). The possible higher levels of cytoplasmic mtrRNAs in inner cells of late preimplantation embryos are in parallel with the cell lineage allocation effects of $16 \mathrm{~S}$ mtrRNA. It is possible that the expression and distribution of mtrRNAs is related to unknown mitochondrial activities in early embryos. MtrRNAs are important components of mitochondrial ribosomes for mitochondrial protein synthesis. Despite the dormant oxidative phosphorylation activity of mitochondria, mitochondrial protein synthesis remains active in early embryos (Piko \& Chase 1973, Cascio \& Wassarman 1981). One possible functional consequence of the cytoplasmic localization of mtrRNAs can be the participation of mitochondrial ribosomes, which are more close to prokaryotic ribosomes than to its eukaryotic cytoplasmic counterparts (Amunts et al. 2014), in the synthesis of specific subsets of mRNAs that may play crucial roles during pre-implantation embryogenesis (Amikura et al. 2001). Intriguingly, latest studies showed that mitochondrial genome contains short ORFs (sORFs) that reside within mtrRNA's genetic loci, which encode short polypeptides that are translated following the export of mtrRNAs (Hashimoto et al. 2001, Lee et al. 2015). These polypeptides (the 24-aa long Humanin from $165 \mathrm{mtrRNA}$ and the 16-aa long MOTS-C from $12 S \mathrm{mtr} R N A$ ) are biologically functional and elicit critical roles in regulating insulin sensitivity and maintaining metabolic homeostasis in neuronal and skeletal muscle cells (Hashimoto et al. 2001, Muzumdar et al. 2009, Lee et al. 2015). It will be interesting to fully elucidate whether mtrRNAs regulate cell lineage allocations during early embryogenesis via RNA-mediated or alternative mechanisms and how mitochondrial genome participates in the process.

The mechanisms of cell fate determination regulated by early embryonic events during embryogenesis have been under intensive investigation (Rossant \& Tam 2009). It is known that blastomeres in early embryos possess developmental plasticity during experimental manipulations. Although molecular alterations within isolated embryonic cells have not been examined, the adaptive nature of embryos suggests that mammalian pre-implantation embryogenesis is a subject of more complicated regulation than previously anticipated (Zernicka-Goetz et al. 2009). During microinjection experiments, altered $16 \mathrm{~S}$ mtrRNA levels influenced cell lineage allocations of descendent in the blastocyst.
Although it is not clear whether effects brought by alterations of mtrRNAs will elicit in a stoichiometric or dose-dependent manner, statistical analyses showed that altered 16S mtrRNA levels brought significant changes to cell lineage allocations of descendent. Specific cell lineage markers (such as NANOG and CDX2), however, maintained constant within respective positions of embryonic cells, even when progeny cells of injected blastomeres were deviated to either ICM or TE. This suggests that $16 \mathrm{~S}$ mtrRNA may participate in the regulation of cell allocations prior to cell lineage specific gene expression (Nance 2014, Rayon et al. 2014). Recent genome-wide gene expression analyses on early embryonic cells in both mouse and $C$. elegans indicated a bi-facet model of cell lineage determination, in which an earlier stochastic heterogeneous geneexpression stage when cells appear non-distinguishable is followed by a late reinforced homogeneous geneexpression stage when cells gain respective molecular signatures (Ohnishi et al. 2014, Du et al. 2015). These suggested that perhaps earlier events preluding more definitive cell-lineage determination pathways are regulated by post-transcriptional mechanisms, involving various RNA species and RNA binding proteins. The correlation between mtrRNAs and cell lineage allocations in the blastocyst indicates that differentially expressed molecules, such as mtrRNAs, are functionally involved in the regulation of cell fate determination at the very beginning of animal development. Future research is required to elucidate the molecular mechanisms that govern the partitioning of mtrRNAs and how they regulate the cell lineage allocation during mouse pre-implantation embryogenesis.

\section{Supplementary data}

This is linked to the online version of the paper at http://dx.doi. org/10.1530/REP-15-0301.

\section{Declaration of interest}

The authors declare that there is no conflict of interest that could be perceived as prejudicing the impartiality of the research reported.

\section{Funding}

This work was supported by the Key Technologies R\&D Program, Ministry of Science and Technology, China (2010CB945402, 2007CB947504), the National Nature Science Foundation of China (30871403, 81561138001), and partly supported by the Chinese Academy of Sciences.

\section{Acknowledgements}

We would like to thank Xingguo Liu and Xiaodong Shu for sharing reagents; Hongwen Pang for the help with using ZEN 
2010 software. We would like to thank David Albertini for the kind suggestion on the experiments of hormonal effects on mice. We would also like to thank the anonymous reviewers for their valuable comments.

\section{References}

Amikura R, Kashikawa M, Nakamura A \& Kobayashi S 2001 Presence of mitochondria-type ribosomes outside mitochondria in germ plasm of Drosophila embryos. PNAS 98 9133-9138. (doi:10.1073/pnas. 171286998)

Amunts A, Brown A, Bai XC, Llacer JL, Hussain T, Emsley P, Long F, Murshudov G, Scheres SH \& Ramakrishnan V 2014 Structure of the yeast mitochondrial large ribosomal subunit. Science 343 1485-1489. (doi:10.1126/science.1249410)

Antczak M \& Van Blerkom J 1997 Oocyte influences on early development: the regulatory proteins leptin and STAT3 are polarized in mouse and human oocytes and differentially distributed within the cells of the preimplantation stage embryo. Molecular Human Reproduction 3 1067-1086. (doi:10.1093/molehr/3.12.1067)

Attardi G \& Attardi B 1968 Mitochondrial origin of membrane-associated heterogeneous RNA in HeLa cells. PNAS 61 261-268. (doi:10.1073/ pnas.61.1.261)

Burton A, Muller J, Tu S, Padilla-Longoria P, Guccione E \& TorresPadilla ME 2013 Single-cell profiling of epigenetic modifiers identifies PRDM14 as an inducer of cell fate in the mammalian embryo. Cell Reports 5 687-701. (doi:10.1016/j.celrep.2013.09.044)

Cascio SM \& Wassarman PM 1981 Program of early development in the mammal: synthesis of mitochondrial proteins during oogenesis and early embryogenesis in the mouse. Developmental Biology 83 166-172. (doi:10.1016/S0012-1606(81)80019-X)

Cree LM, Samuels DC, de Sousa Lopes SC, Rajasimha HK, Wonnapinij P, Mann JR, Dahl HH \& Chinnery PF 2008 A reduction of mitochondrial DNA molecules during embryogenesis explains the rapid segregation of genotypes. Nature Genetics 40 249-254. (doi:10.1038/ng.2007.63)

Dard N, Louvet-Vallee S, Santa-Maria A \& Maro B 2004 Phosphorylation of ezrin on threonine T567 plays a crucial role during compaction in the mouse early embryo. Developmental Biology 271 87-97. (doi:10.1016/ j.ydbio.2004.03.024)

Du Z, Santella A, He F, Shah PK, Kamikawa Y \& Bao Z 2015 The regulatory landscape of lineage differentiation in a metazoan embryo. Developmental Cell 34 592-607. (doi:10.1016/j.devcel.2015.07.014)

Ducibella T \& Anderson E 1975 Cell shape and membrane changes in the eight-cell mouse embryo: pre-requisities for morphogenesis of the blastocyst. Developmental Biology 47 45-58. (doi:10.1016/00121606(75)90262-6)

Fujimori T, Kurotaki Y, Miyazaki J \& Nabeshima Y 2003 Analysis of cell lineage in two- and four-cell mouse embryos. Development 130 5113-5122. (doi:10.1242/dev.00725)

Gardner RL 1997 The early blastocyst is bilaterally symmetrical and its axis of symmetry is aligned with the animal-vegetal axis of the zygote in the mouse. Development 124 289-301.

Gardner RL 2001 Specification of embryonic axes begins before cleavage in normal mouse development. Development 128 839-847.

Guo G, Huss M, Tong GQ, Wang C, Li Sun L, Clarke ND \& Robson P 2010 Resolution of cell fate decisions revealed by single-cell gene expression analysis from zygote to blastocyst. Developmental Cell 18 675-685. (doi:10.1016/j.devcel.2010.02.012)

Hashimoto Y, Niikura T, Tajima H, Yasukawa T, Sudo H, Ito Y, Kita Y, Kawasumi M, Kouyama K, Doyu M et al. 2001 A rescue factor abolishing neuronal cell death by a wide spectrum of familial Alzheimer's disease genes and A $\beta$. PNAS 98 6336-6341. (doi:10.1073/pnas.101133498)

lida T \& Kobayashi S 1998 Essential role of mitochondrially encoded large rRNA for germ-line formation in Drosophila embryos. PNAS 95 11274-11278. (doi:10.1073/pnas.95.19.11274)

Jedrusik A, Parfitt DE, Guo G, Skamagki M, Grabarek JB, Johnson MH, Robson P \& Zernicka-Goetz M 2008 Role of Cdx2 and cell polarity in cell allocation and specification of trophectoderm and inner cell mass in the mouse embryo. Genes and Development 22 2692-2706. (doi:10. 1101/gad.486108)
Jefferson WN \& Williams CJ 2012 Early mouse embryo asymmetry. Molecular Reproduction and Development 79 433. (doi:10.1002/mrd. 22050)

Johnson MH 2009 From mouse egg to mouse embryo: polarities, axes, and tissues. Annual Review of Cell and Developmental Biology 25 483-512. (doi:10.1146/annurev.cellbio.042308.113348)

Johnson MH \& Ziomek CA 1981 The foundation of two distinct cell lineages within the mouse morula. Cell 24 71-80. (doi:10.1016/0092-8674 (81)90502-X)

Johnstone O \& Lasko P 2001 Translational regulation and RNA localization in Drosophila oocytes and embryos. Annual Review of Genetics 35 365-406. (doi:10.1146/annurev.genet.35.102401.090756)

Kimble J \& Crittenden SL 2007 Controls of germline stem cells, entry into meiosis, and the sperm/oocyte decision in Caenorhabditis elegans. Annual Review of Cell and Developmental Biology 23 405-433. (doi:10. 1146/annurev.cellbio.23.090506.123326)

Kobayashi S, Amikura R \& Okada M 1993 Presence of mitochondrial large ribosomal RNA outside mitochondria in germ plasm of Drosophila melanogaster. Science 260 1521-1524. (doi:10.1126/science.7684857)

Kobayashi S, Amikura R \& Mukai M 1998 Localization of mitochondrial large ribosomal RNA in germ plasm of Xenopus embryos. Current Biology 8 1117-1120. (doi:10.1016/S0960-9822(98)70466-X)

Krisher RL \& Prather RS 2012 A role for the Warburg effect in preimplantation embryo development: metabolic modification to support rapid cell proliferation. Molecular Reproduction and Development 79 311-320. (doi:10.1002/mrd.22037)

Kurotaki Y, Hatta K, Nakao K, Nabeshima Y \& Fujimori T 2007 Blastocyst axis is specified independently of early cell lineage but aligns with the ZP shape. Science 316 719-723. (doi:10.1126/science.1138591)

Lee MT, Bonneau AR \& Giraldez AJ 2014 Zygotic genome activation during the maternal-to-zygotic transition. Annual Review of Cell and Developmental Biology 30 581-613. (doi:10.1146/annurev-cellbio100913-013027)

Lee C, Zeng J, Drew BG, Sallam T, Martin-Montalvo A, Wan J, Kim SJ, Mehta H, Hevener AL, de Cabo R et al. 2015 The mitochondrial-derived peptide MOTS-c promotes metabolic homeostasis and reduces obesity and insulin resistance. Cell Metabolism 21 443-454. (doi:10.1016/j. cmet.2015.02.009)

Li R \& Albertini DF 2013 The road to maturation: somatic cell interaction and self-organization of the mammalian oocyte. Nature Reviews. Molecular Cell Biology 14 141-152. (doi:10.1038/nrm3531)

Li L, Baibakov B \& Dean J 2008 A subcortical maternal complex essential for preimplantation mouse embryogenesis. Developmental Cell 15 416-425. (doi:10.1016/j.devcel.2008.07.010)

Maniataki E \& Mourelatos Z 2005 Human mitochondrial tRNAMet is exported to the cytoplasm and associates with the Argonaute 2 protein. RNA 11 849-852. (doi:10.1261/rna.2210805)

Mishra P \& Chan DC 2014 Mitochondrial dynamics and inheritance during cell division, development and disease. Nature Reviews. Molecular Cell Biology 15 634-646. (doi:10.1038/nrm3877)

Motosugi N, Bauer T, Polanski Z, Solter D \& Hiiragi T 2005 Polarity of the mouse embryo is established at blastocyst and is not prepatterned. Genes and Development 19 1081-1092. (doi:10.1101/gad.1304805)

Muzumdar RH, Huffman DM, Atzmon G, Buettner C, Cobb LJ, Fishman S, Budagov T, Cui L, Einstein FH, Poduval A et al. 2009 Humanin: a novel central regulator of peripheral insulin action. PLOS ONE 4 e6334. (doi:10.1371/journal.pone.0006334)

Nance J 2014 Getting to know your neighbor: cell polarization in early embryos. Journal of Cell Biology 206 823-832. (doi:10.1083/jcb. 201407064)

Ninomiya Y \& Ichinose S 2007 Subcellular distribution of mitochondrial ribosomal RNA in the mouse oocyte and zygote. PLOS ONE 2 e1241. (doi:10.1371/journal.pone.0001241)

Niwa H, Toyooka Y, Shimosato D, Strumpf D, Takahashi K, Yagi R \& Rossant J 2005 Interaction between Oct3/4 and $\mathrm{Cdx} 2$ determines trophectoderm differentiation. Cell 123 917-929. (doi:10.1016/j.cell. 2005.08.040)

Ogawa M, Amikura R, Akasaka K, Kinoshita T, Kobayashi S \& Shimada H 1999 Asymmetrical distribution of mitochondrial rRNA into small micromeres of Sea urchin embryos. Zoological Science 16 445-451. (doi:10.2108/zsj.16.445) 
Ohnishi $\mathrm{Y}$, Huber W, Tsumura A, Kang $\mathrm{M}$, Xenopoulos P, Kurimoto $\mathrm{K}$, Oles AK, Arauzo-Bravo MJ, Saitou M, Hadjantonakis AK et al. 2014 Cell-to-cell expression variability followed by signal reinforcement progressively segregates early mouse lineages. Nature Cell Biology $\mathbf{1 6}$ 27-37. (doi:10.1038/ncb2881)

Piko L \& Chase DG 1973 Role of the mitochondrial genome during early development in mice. Effects of ethidium bromide and chloramphenicol. Journal of Cell Biology 58 357-378. (doi:10.1083/jcb.58. 2.357)

Piko L \& Taylor KD 1987 Amounts of mitochondrial DNA and abundance of some mitochondrial gene transcripts in early mouse embryos. Developmental Biology 123 364-374. (doi:10.1016/0012-1606(87)90395-2)

Piotrowska K \& Zernicka-Goetz M 2001 Role for sperm in spatial patterning of the early mouse embryo. Nature 409 517-521. (doi:10.1038/ 35054069)

Piotrowska K, Wianny F, Petersen RA \& Zernicka-Goetz M 2001 Blastomeres arising from the first cleavage division have distinguishable fates in normal mouse development. Development 128 3739-3748.

Piotrowska-Nitsche K \& Zernicka-Goetz M 2005 Spatial arrangement of individual 4-cell stage blastomeres and the order in which they are generated correlate with blastocyst pattern in the mouse embryo. Mechanisms of Development 122 487-500. (doi:10.1016/j.mod.2004. 11.014)

Plachta N, Bollenbach T, Pease S, Fraser SE \& Pantazis P 2011 Oct4 kinetics predict cell lineage patterning in the early mammalian embryo. Nature Cell Biology 13 117-123. (doi:10.1038/ncb2154)

Politi Y, Gal L, Kalifa Y, Ravid L, Elazar Z \& Arama E 2014 Paternal mitochondrial destruction after fertilization is mediated by a common endocytic and autophagic pathway in Drosophila. Developmental Cell 29 305-320. (doi:10.1016/j.devcel.2014.04.005)

Rayon T, Menchero S, Nieto A, Xenopoulos P, Crespo M, Cockburn K, Canon S, Sasaki H, Hadjantonakis AK, de la Pompa JL et al. 2014 Notch and hippo converge on $\mathrm{cdx} 2$ to specify the trophectoderm lineage in the mouse blastocyst. Developmental Cell 30 410-422. (doi:10.1016/j. devcel.2014.06.019)

Rossant J \& Tam PP 2009 Blastocyst lineage formation, early embryonic asymmetries and axis patterning in the mouse. Development $\mathbf{1 3 6}$ 701-713. (doi:10.1242/dev.017178)

Sanfins A, Lee GY, Plancha CE, Overstrom EW \& Albertini DF 2003 Distinctions in meiotic spindle structure and assembly during in vitro and in vivo maturation of mouse oocytes. Biology of Reproduction 69 2059-2067. (doi:10.1095/biolreprod.103.020537)
Schulz LC \& Roberts RM 2011 Dynamic changes in leptin distribution in the progression from ovum to blastocyst of the pre-implantation mouse embryo. Reproduction 141 767-777. (doi:10.1530/REP-10-0532)

St Johnston D \& Ahringer J 2010 Cell polarity in eggs and epithelia: parallels and diversity. Cell 141 757-774. (doi:10.1016/j.cell.2010.05.011)

Tabansky I, Lenarcic A, Draft RW, Loulier K, Keskin DB, Rosains J, RiveraFeliciano J, Lichtman JW, Livet J, Stern JN et al. 2013 Developmental bias in cleavage-stage mouse blastomeres. Current Biology 23 21-31. (doi:10.1016/j.cub.2012.10.054)

Tarkowski AK, Suwinska A, Czolowska R \& Ozdzenski W 2010 Individual blastomeres of 16- and 32-cell mouse embryos are able to develop into foetuses and mice. Developmental Biology 348 190-198. (doi:10.1016/ j.ydbio.2010.09.022)

Torres-Padilla ME, Parfitt DE, Kouzarides T \& Zernicka-Goetz M 2007 Histone arginine methylation regulates pluripotency in the early mouse embryo. Nature 445 214-218. (doi:10.1038/nature05458)

Weaver S, Dube S, Mir A, Qin J, Sun G, Ramakrishnan R, Jones RC \& Livak KJ 2010 Taking qPCR to a higher level: analysis of CNV reveals the power of high throughput qPCR to enhance quantitative resolution. Methods 50 271-276. (doi:10.1016/j.ymeth.2010.01.003)

Whitaker M 2006 Calcium at fertilization and in early development. Physiological Reviews 86 25-88. (doi:10.1152/physrev.00023.2005)

Yamashita S, Katsumata O \& Okada Y 2009 Establishment of a standardized post-embedding method for immunoelectron microscopy by applying heat-induced antigen retrieval. Journal of Electron Microscopy $\mathbf{5 8}$ 267-279. (doi:10.1093/jmicro/dfp017)

Zeng FY, Baldwin DA \& Schultz RM 2004 Transcript profiling during pre-implantation mouse development. Developmental Biology 272 483-496. (doi:10.1016/j.ydbio.2004.05.018)

Zernicka-Goetz M, Morris SA \& Bruce AW 2009 Making a firm decision: multifaceted regulation of cell fate in the early mouse embryo. Nature Reviews. Genetics 10 467-477. (doi:10.1038/nrg2564)

Zhang QF, Cui JM, Huang XJ, Zheng HY, Huang JC, Fang L, Li KP \& Zhang JQ 2004 The life cycle of SARS coronavirus in Vero E6 cells. Journal of Medical Virology 74 332-337. (doi:10.1002/jmv.20095)

Received 29 June 2015

First decision 24 July 2015

Revised manuscript received 6 January 2016

Accepted 13 January 2016 